\title{
Paramodulation with Non-Monotonic Orderings and Simplification
}

\author{
Miquel Bofill · Albert Rubio
}

Received: date / Accepted: date

\begin{abstract}
Ordered paramodulation and Knuth-Bendix completion are known to remain complete when using non-monotonic orderings. However, these results do not imply the compatibility of the calculus with essential redundancy elimination techniques such as demodulation, i.e., simplification by rewriting, which constitute the primary mode of computation in most successful automated theorem provers.

In this paper we present a complete ordered paramodulation calculus for non-monotonic orderings which is compatible with powerful redundancy notions including demodulation, hence strictly improving the previous results and making the calculus more likely to be used in practice.

As a side effect, we obtain a Knuth-Bendix completion procedure compatible with simplification techniques, which can be used for finding, whenever it exists, a convergent term rewrite system for a given set of equations and a (possibly non-totalizable) reduction ordering.
\end{abstract}

Keywords automated theorem proving - equational reasoning - ordered paramodulation Knuth-Bendix completion

This work has been partially supported by the Spanish MEC/MICINN under grants TIN 2008-04547 and TIN 2010-68093-C02-01. A preliminary version of this work was presented at the IJCAR 2004 conference (Bofill and Rubio (2004)).

\section{Miquel Bofill}

Universitat de Girona

Dept. Informàtica i Matemàtica Aplicada

Campus Montilivi, Ed. P4

E-17071 Girona, Spain

Tel.: +34-972418838

Fax: +34-972418792

E-mail: mbofill@ima.udg.edu

Albert Rubio

Universitat Politècnica de Catalunya

Dept. Llenguatges i Sistemes Informàtics

C/ Jordi Girona, 1-3

E-08034 Barcelona, Spain

E-mail: rubio@1si.upc.edu 


\section{Introduction}

Knuth-Bendix-like completion techniques and their extensions to ordered paramodulation for first-order clauses are among the most successful methods for automated deduction with equality (Bachmair and Ganzinger (1998); Nieuwenhuis and Rubio (2001)). For many years, all known completeness results for Knuth-Bendix completion and ordered paramodulation required the term ordering $\succ$ to be well-founded, monotonic and total (or extendable to a total ordering) on ground terms (Hsiang and Rusinowitch (1991); Bachmair et al (1986); Bachmair and Dershowitz (1994); Bachmair and Ganzinger (1994)). In Bofill et al (1999, 2003), the monotonicity requirement was dropped and well-foundedness and the subterm property were shown to be sufficient for ensuring refutation completeness of ordered paramodulation (notice that any such ordering can be totalized without losing these two properties). And in Bofill and Rubio (2002, 2009) it was shown that well-foundedness of the ordering suffices for completeness of ordered paramodulation for Horn clauses, i.e., the subterm property can be dropped as well.

Apart from its theoretical value, these results have several potential applications in contexts where the usual requirements are too strong. For example, in deduction modulo built-in equational theories $E$, where $E$-compatibility of the ordering (i.e., $s={ }_{E} s^{\prime} \succ t^{\prime}={ }_{E} t$ implies $s \succ t$ ) is needed, finding $E$-compatible orderings fulfilling the required properties is extremely complex or even impossible. For instance, when $E$ contains an idempotency axiom $f(x, x) \simeq x$, no total $E$-compatible reduction ordering exists: if $s \succ t$, then by monotonicity one should have $f(s, s) \succ f(s, t)$ which, by $E$-compatibility, implies $s \succ f(s, t)$ and hence non-well-foundedness. Therefore, the techniques for dropping ordering requirements, among other applications, open the door to deduction modulo many more classes of equational theories.

Another source of the interest in dropping ordering requirements is that, in many cases, it is not clear if a particular ordering will be good (e.g. for reducing the search space) in some given problem. Hence, broadening the range of usable orderings can be helpful in practice. Indeed, there exist examples of problems for which (unfailing) Knuth-Bendix style procedures only terminate if we choose a reduction ordering which is not extendable to a total one.

\section{Example ${ }^{1}$}

Consider the closure under standard Knuth-Bendix completion of the following two rules:

$$
\begin{aligned}
h(x) & \rightarrow f g(x) \\
h f g(x) & \rightarrow f f g(x)
\end{aligned}
$$

Between these two rules there is a single critical pair since $h f g(z)$ can be rewritten into both $f f g(z)$ and $f g f g(z)$. Therefore in a Knuth-Bendix completion the equation

$$
f f g(z) \simeq f g f g(z)
$$

should be added. If we work with a well-founded and monotonic total ordering then the previous equation is necessarily oriented into

$$
f g f g(x) \rightarrow f f g(x)
$$

\footnotetext{
1 We thank Christopher Lynch for providing us with this example.
} 
(Notice that, otherwise, we will contradict either well-foundedness or monotonicity or totality on ground terms, because if $f f g(x) \succ f g f g(x)$ then $f g(x)$ and $g f g(x)$ must be incomparable in any well-founded and monotonic extension of $\succ$ :

- If $f g(x) \succ g f g(x)$ then it contradicts the subterm relation, and then by monotonicity we can get an infinite decreasing sequence $f g(x) \succ g f g(x) \succ g g f g(x) \succ$ $\ldots$

- If $g f g(x) \succ f g(x)$ then by monotonicity we have $f g f g(x) \succ f f g(x)$, leading to reflexivity and hence to the existence of an infinite decreasing sequence.)

Unfortunately, with the "right" orientation $f g f g(x) \rightarrow f f g(x)$, standard Knuth-Bendix completion would generate an infinite set of rules of the form

$$
f g f^{n} g(x) \rightarrow f^{n+1} g(x) \quad \text { with } n \geq 1 .
$$

However, if we take the unusual orientation, i.e.,

$$
f f g(x) \rightarrow f g f g(x),
$$

the system is already closed, i.e., standard Knuth-Bendix completion would generate only the additional rule

$$
f f g(x) \rightarrow f g f g(x)
$$

Recall that, as shown above, with this orientation the ordering cannot be extended to a total one. Notice also that, even if we compute inferences with left-hand sides into righthand sides (in the sense of ordered paramodulation) the system would be closed after adding $f f g(x) \rightarrow f g f g(x)$ to the set.

By now we have motivated the interest of dropping ordering requirements. However, ordered strategies are useful in practice only if compatibility with redundancy is shown. Simplification of formulae and elimination of redundant formulae are essential components of automated theorem provers. In fact, in most successful automated theorem provers, simplification is the primary mode of computation, whereas prolific deduction rules are used only sparingly.

In this direction, here we present a paramodulation based calculus which strictly improves the one in Bofill et al (2003), for which refutation completeness was shown for non-totalizable reduction orderings, but compatibility with simplification techniques was left open. On the one hand, regarding the amount of inferences needed to be performed, the inference system presented here is essentially the same as the one in Bofill et al (2003), but, on the other hand, this calculus is compatible with powerful redundancy notions which include demodulation, i.e., simplification by rewriting. Also, as in Bofill et al (2003), we can apply our results to obtain a Knuth-Bendix style completion procedure, but in this case compatible with simplification techniques. This procedure can be used for finding, whenever it exists, a convergent TRS for a given set of equations and a (possibly non-totalizable) reduction ordering.

In our calculus, it is assumed that equations are oriented w.r.t. a possibly non-monotonic ordering $\succ$ which is an extension of a known reduction ordering $\succ_{r}$. As in Bofill et al (2003), the ordering $\succ$ is required to (i) be well-founded, (ii) fulfill the subterm property and (iii) be total on ground terms. In the case that $\succ$ is defined on first order terms, we require it to be stable under substitutions. As shown in Bofill et al (2003), every reduction ordering can be extended to a total ordering fulfilling these properties (at the expense, however, of possibly losing monotonicity). 
We show that some redundancy notions w.r.t. $\succ$ (in particular, w.r.t. the reduction ordering $\succ_{r}$ included in $\succ$ ) can be applied in this framework while keeping refutation completeness. In the ground case, if all equations involved in the saturation ${ }^{2}$ process turn out to be orientable with $\succ_{r}$, then demodulation can be fully applied. But, in general, in order to preserve refutation completeness we must impose some limitations to the terms that can be simplified. During our saturation process, we mark out some subterms of the clauses, which become blocked for demodulation (technically, the marked subterms are interpreted as variables for redundancy purposes). Roughly, the idea is to mark out the terms that are introduced in the conclusion of an inference that are not smaller w.r.t. $\succ_{r}$ than some term in the maximal premise. Also, some variables need to be marked when performing redundancy steps (as explained in Section 5.5). In fact, as shown in Example 6 of Section 5.5, refutation completeness can be lost when applying paramodulation w.r.t. a non-monotonic ordering $\succ$ extending a reduction ordering $\succ_{r}$, together with demodulation w.r.t. $\succ_{r}$, if no blockings are introduced at all.

The reason for adding the blockings also has technical roots, coming from the technique used in the completeness proof, which is based on the model generation technique of Bachmair and Ganzinger (1994) and its variant used in Bofill et al (2003). In the latter, in contrast to the former, the ordering used for orienting the equations and the ordering used for induction in the completeness proof do not need to coincide. Concretely, equations are oriented w.r.t. a (possibly) non-monotonic ordering $\succ$, whereas completeness is proved by induction w.r.t. a rewrite (and hence monotonic) relation $\stackrel{+}{\rightarrow}_{R}$, where $R$ is a limit ground rewrite system, built up from a subset of equations in the closure. The fact that these two orderings do not need to coincide is the key for the completeness proof of ordered paramodulation with non-monotonic orderings given in Bofill et al (2003). Now, if we want to add redundancy notions, the first natural choice is to define them w.r.t. the ordering $\succ$ that we use in the ordered paramodulation inference rules. However, since completeness is proved by induction w.r.t. $\stackrel{+}{\rightarrow}_{R}$, redundancy notions should be defined w.r.t. $\stackrel{+}{\rightarrow}_{R}$ as well. Unfortunately, $\stackrel{+}{\rightarrow}_{R}$ is unknown during the saturation process, and moreover it is not clear at all how it can be approximated sufficiently. This is why in Bofill et al (2003) it was left open to what extent demodulation could be applied in this setting (although some practical redundancy notions such as tautology deletion and subsumption were shown to preserve completeness).

As said, here our aim is mainly to add demodulation w.r.t. the reduction ordering $\succ_{r}$, which is included in the (possibly non-monotonic) extension $\succ$ used to orient the equations. The idea for doing that is to find a well-founded ordering $\succ_{R}$ which, roughly, combines $\succ$ with $\stackrel{+}{\rightarrow}_{R}$, and then prove completeness by induction w.r.t. $\succ_{R}$. The problem is that, although $\succ_{r}$ is a well-founded and monotonic ordering and $R$ is a terminating TRS whose rules are included in a well-founded extension $\succ$ of $\succ_{r}$, the relation $\rightarrow_{R} \cup \succ$ is not well-founded in general and, in fact, $\stackrel{+}{\rightarrow}_{R}$ can even contradict $\succ$. Take, e.g., $f(b) \succ_{r} f(a)$ and a well-founded extension $\succ$ of $\succ_{r}$ such that $f(b) \succ f(a) \succ a \succ b$. Then possibly $a \rightarrow b \in R$ (since the rules in $R$ are oriented w.r.t. $\succ$ ) and hence the infinite sequence $f(a) \rightarrow_{R} f(b) \succ f(a) \cdots$ can be built.

The idea to circumvent this non-well-foundedness problem is to block the terms that are introduced by a rewriting step with $R$ that is not included in $\succ$. In the previous example, since $f(b) \succ f(a)$, then we rewrite $f(a)$ with $a \rightarrow b$ into $f(b)$ with $b$ blocked, and we consider that $f(b)$ with $b$ blocked is no longer greater than $f(a)$ w.r.t. $\succ$. Then, roughly, if the comparisons with $\succ$ do not take into account the blocked terms, we can combine $\succ$

\footnotetext{
2 The saturation of a set of clauses $S$ amounts to the closure of $S$ under the inference system up to redundancy.
} 
with $\rightarrow_{R}$ in a well-founded way. Altogether, it gives us some amount of redundancy w.r.t. $\succ$, while preserving refutation completeness.

A convenient way to represent terms with blocked positions is by means of superindexed subterms, also called marked terms. For example $f\left(b^{x}\right)$, where $x$ is a variable, denotes the term $f(b)$ where the subterm $b$ is blocked. Then, although for performing inferences $f\left(b^{x}\right)$ still corresponds to the term $f(b)$, for the redundancy notions it will be seen as $f(x)$, with a blocked term $b$ in $x$. In this context $f\left(b^{x}\right)$ cannot be simplified with $f(b) \rightarrow f(a)$, since $f(b)$ does not match $f(x)$.

Marked terms resemble the term closures of Bachmair et al (1995). However, their semantics is fairly different: here the blockings only have effect on the redundancy notions since, although marked terms are seen as variables for redundancy purposes, the ordered paramodulation inferences are applied at both blocked ${ }^{3}$ and unblocked positions. The terms to be marked will come mainly from non-reductive inferences. Therefore, the more equations can be handled by the reduction ordering, the less terms will be blocked in the conclusions of the inferences, and the more redundancy will be possible.

The rest of the paper is structured as follows. Preliminaries are presented in Section 2. Marked terms and orderings on marked terms are defined in Section 3. In Section 4, our calculus, including the notion of redundancy of inferences, is presented for deduction with sets of equations. It is extended to general first order clauses in Section 5 where, moreover, saturation derivations including redundancy of clauses are considered. In Section 6 we show how, from our results, a Knuth-Bendix completion procedure for finding convergent TRSs can be obtained. In Section 7 we comment on some experiments. In Section 8 we describe a way of defining the kind of non-monotonic orderings that we need in this paper, which could be easily automated. Finally in Section 9 we conclude.

\section{Preliminaries}

\subsection{Terms, Equations and (Equality) Clauses}

We use the standard definitions of Nieuwenhuis and Rubio (2001). $T(\mathscr{F}, \mathscr{X})(T(\mathscr{F}))$ is the set of (ground) terms over a set of symbols $\mathscr{F}$ and a denumerable set of variables $\mathscr{X}$ (over $\mathscr{F})$. By $\operatorname{Var}(t)$ we denote the set of variables occurring in a term $t$. The subterm of $t$ at position $p$ is denoted by $\left.t\right|_{p}$, the result of replacing $\left.t\right|_{p}$ by $s$ in $t$ is denoted by $t[s]_{p}$, and syntactic equality of terms is denoted by $\equiv$. A context $t[]_{p}$ is a term $t$ with a hole at a distinguished position $p$. A substitution is a partial mapping from variables to terms. The application of a substitution $\sigma$ to a term $t$ is denoted by $t \sigma$. The composition of two substitutions $\sigma_{1}$ and $\sigma_{2}$, denoted by juxtaposition, is defined as the composition of two functions, that is, $t \sigma_{1} \sigma_{2}=\left(t \sigma_{1}\right) \sigma_{2}$. The substitution $\left.\sigma\right|_{A}$ is the substitution $\sigma$ restricted to the variables of $A$.

An equation is a multiset of terms $\{s, t\}$, denoted $s \simeq t$ or, equivalently, $t \simeq s$. A firstorder clause is a pair of finite multisets of equations $\Gamma$ (the antecedent) and $\Delta$ (the succedent), denoted by $\Gamma \rightarrow \Delta$. Equations in $\Gamma$ are called negative literals and equations in $\Delta$ are called positive literals. A Horn clause is a clause with at most one positive literal. The empty clause $\square$ is a clause where both $\Gamma$ and $\Delta$ are empty.

\footnotetext{
3 All the positions under a marked term are considered as blocked for redundancy.
} 


\subsection{Terms and (Rewrite) Relations}

If $\rightarrow$ is a binary relation, then $\leftarrow$ is its inverse, $\leftrightarrow$ is its symmetric closure, $\stackrel{+}{\rightarrow}$ is its transitive closure and $\stackrel{*}{\rightarrow}$ is its reflexive-transitive closure. If $s \stackrel{*}{\rightarrow} t$ and there is no $t^{\prime}$ such that $t \rightarrow t^{\prime}$ then $t$ is called irreducible and a normal form of $s$ (w.r.t. $\rightarrow$ ). A relation $\rightarrow$ is well-founded or terminating if there exists no infinite sequence $s_{1} \rightarrow s_{2} \rightarrow \cdots$. The composition of two relations is indicated by $\circ$. Thus $s \stackrel{*}{\leftarrow} \circ \stackrel{*}{\rightarrow} t$ if there is an element $r$ such that $s \stackrel{*}{\leftarrow} r$ and $r \stackrel{*}{\rightarrow} t$. A relation $\rightarrow$ is confluent or Church-Rosser if the relation $\stackrel{*}{\leftarrow} \circ \stackrel{*}{\rightarrow}$ is contained in $\stackrel{*}{\rightarrow} \circ \stackrel{*}{\leftarrow}$. A relation $\rightarrow$ on terms is monotonic if $s \rightarrow t$ implies $u[s]_{p} \rightarrow u[t]_{p}$ for all terms $s, t$ and $u$ and positions $p$. An equivalence relation is a reflexive, symmetric and transitive binary relation. A congruence is a monotonic equivalence relation.

A rewrite rule is an ordered pair of terms $(s, t)$, written $s \rightarrow t$, and a set of rewrite rules $R$ is a term rewrite system (TRS). The rewrite relation with $R$ on $T(\mathscr{F}, \mathscr{X})$, denoted by $\rightarrow_{R}$, is the smallest monotonic relation such that for all rules $l \rightarrow r$ in $R$, and substitutions $\sigma, l \sigma \rightarrow_{R}$ $r \sigma$. If $s \rightarrow_{R} t$ then we say that $s$ rewrites into $t$ with $R$. Obtaining a normal form of a term $t$ by rewriting with a TRS $R$ is called a normalization of $t$ with respect to $R$ and is denoted by $t \downarrow_{R}$. A TRS $R$ is called terminating, confluent, etc. if $\rightarrow_{R}$ is. It is called convergent if it is confluent and terminating. The congruence $\stackrel{*}{\leftrightarrow} R$ defines an equality Herbrand interpretation where $\simeq$ is interpreted by $s \simeq t$ iff $s \stackrel{*}{\leftrightarrow}_{R} t$. Such an interpretation will be denoted by $R^{*}$.

A (strict partial) ordering $\succ$ on $T(\mathscr{F}, \mathscr{X})$ is an irreflexive and transitive binary relation. It is stable under substitutions if $s \succ t$ implies $s \sigma \succ t \sigma$ for all substitutions $\sigma$. Monotonic orderings that are stable under substitutions are called rewrite orderings. A reduction ordering is a well-founded rewrite ordering.

A rewrite system terminates if, and only if, its rules are contained in a reduction ordering. In fact, if $R$ is a terminating TRS, then $\stackrel{+}{\rightarrow}_{R}$ is a reduction ordering. However in restricted cases checking the rules with a well-founded ordering is enough:

Lemma 1 (Bofill et al (2003)) Let $\succ$ be a well-founded ordering, and let $R$ be a ground TRS such that, for all $l \rightarrow r$ in $R, l \succ r$ and $r$ is irreducible by $R$ at non-topmost positions. Then $R$ is terminating.

A quasi-ordering $\succeq$ is a reflexive and transitive binary relation; the associated equivalence relation $\sim$ is the intersection of $\succeq$ with its inverse; the associated strict partial ordering $\succ$ is their difference. Hence, $\succeq$ is the disjoint union of $\succ$ and $\sim$. We say that a relation $\succ$ is compatible with an equivalence relation $\sim$ if $s \sim s^{\prime} \succ t^{\prime} \sim t$ implies $s \succ t$.

Let $\sim$ be an equivalence relation and $\succ$ an ordering. The multiset extension of $\sim$ is defined as the smallest relation $\sim^{m u l}$ on multisets of elements such that

$$
\emptyset \sim^{m u l} \emptyset
$$

and

$$
S \cup\{s\} \sim^{m u l} S^{\prime} \cup\{t\} \quad \text { if } \quad S \sim^{m u l} S^{\prime} \text { and } s \sim t .
$$

The multiset extension of $\succ$ with respect to $\sim$ is defined as the smallest ordering $\succ^{m u l}$ on multisets of elements such that

$$
M \cup\{s\} \succ^{m u l} N \cup\left\{t_{1}, \ldots, t_{n}\right\} \quad \text { if } \quad M \sim^{m u l} N \text { and } s \succ t_{i} \text { for all } i \in 1 \ldots n .
$$

Sometimes the notation $\succ^{m u l}$ is used without explicitly indicating which is the equivalence relation $\sim$. In these cases $\sim$ is assumed to be the syntactic equality relation. If $\succ$ is a wellfounded ordering on a set $S$ and $\succ$ is compatible with $\sim$, then $\succ^{m u l}$ is a well-founded ordering on finite multisets over $S$ (Dershowitz and Manna (1979)). 
There are some (quasi-)orderings that play a central role in our results (in particular, in the redundancy notions). The non-strict subterm relation is denoted by $\unrhd$, while the strict subterm relation is denoted by $\triangleright$. The subsumption relation, denoted by $\geq$, is a quasiordering defined as $s \geq t$ if $s$ is an instance of $t$, i.e., $s \equiv t \sigma$ for some $\sigma$. The equivalence relation associated with $\geq$ is denoted by $\doteq$. Notice that if $s \doteq t$ then $t$ is a variable renamed version (or a variant) of $s$. The encompassment relation, denoted by $\unrhd$, is a quasi-ordering defined as $s \unrhd t$ if a subterm of $s$ is an instance of $t$, i.e., $s \unrhd t \sigma$ for some $\sigma$; therefore, encompassment is the composition of the subterm and the subsumption relations. It is folk knowledge that, if $\succ$ is a reduction ordering, then the relation $\succ U>$ is well-founded (see, e.g., Dershowitz and Jouannaud (1990)).

It is said that an ordering fulfills the subterm property if $\succ \supseteq \triangleright$. A west ordering (Bofill et al (2003)) is a well-founded ordering on $T(\mathscr{F}, \mathscr{X})$ that fulfills the subterm property and that is total on $T(\mathscr{F})$ (it is called west after well-founded, subterm and total) and is stable under substitutions.

Every well-founded ordering can be totalized on $T(\mathscr{F})$ (Wechler (1992)) and hence every well-founded ordering satisfying the subterm property can be extended to a west ordering. We also have that every reduction ordering can be extended to a west ordering (Bofill et al (2003)).

\section{Marked Terms}

\subsection{Definition}

Marked terms resemble the term closures of Bachmair et al (1995). A marked term, following the definition of Bachmair et al (1995), is a pair $s \cdot \gamma$ consisting of a term $s$ (the skeleton) and an idempotent substitution $\gamma$ from variables to terms. For example, $f(x, g(y)) \cdot\{x \mapsto$ $a, y \mapsto h(z)\}$ is a closure with skeleton $f(x, g(y))$ and substitution $\{x \mapsto a, y \mapsto h(z)\}$. Marked terms are extended in the natural way to equations and clauses. An equation between marked terms is called a marked equation, and a clause on marked equations is called a marked clause.

In order to ease the reading, sometimes we will denote marked terms by superindexing their marked subterms with variables. For example,

$$
f(x, g(y)) \cdot\{x \mapsto a, y \mapsto h(z)\}
$$

will be written as

$$
f\left(a^{x}, g\left(h(z)^{y}\right)\right) .
$$

By Forget $(s \cdot \gamma)$ we denote the term $s \gamma$, e.g.,

$$
\text { Forget }(f(x, g(y)) \cdot\{x \mapsto a, y \mapsto h(z)\})=f(a, g(h(z))) .
$$

This notation is extended to equations and clauses in the usual way.

Given a substitution $\gamma$ of the form $\left\{x_{1} \mapsto t_{1}, \ldots, x_{n} \mapsto t_{n}\right\}$, the domain of $\gamma$, denoted by $\operatorname{Dom}(\gamma)$, is defined as the set of variables $\left\{x_{1}, \ldots, x_{n}\right\}$, and the range of $\gamma$, denoted by $\operatorname{Ran}(\gamma)$, is defined as the set of terms $\left\{t_{1}, \ldots, t_{n}\right\}$. The variables occurring in $\operatorname{Dom}(\gamma)$ are called marking variables. Given a marked term $s \cdot \gamma$, a position $p$ of $s$ is called a marked position if $\left.s\right|_{p}$ is a variable in $\operatorname{Dom}(\gamma)$, i.e., a marking variable. For instance, 1 is a marked position of $h(x, y) \cdot\{x \mapsto a\}$, but 2 is not. 
Given a marked term $s \cdot \gamma$ we can assume, if necessary, that $\operatorname{Dom}(\gamma) \subseteq \operatorname{Var}(s)$, i.e., that the substitution is restricted to the variables occurring in the skeleton to which it applies. Moreover, we assume that no variable in $\operatorname{Dom}(\gamma)$ occurs in $\operatorname{Ran}(\gamma)$. This is because, in our setting, the substitution is only used to identify marked subterms. E.g., $h(x, y) \cdot\{x \mapsto a\}$, written as $h\left(a^{x}, y\right)$, denotes the term $h(a, y)$ where the subterm $a$ is marked (notice that a skeleton can contain both marking and non-marking variables, such as $x$ and $y$ in this example). Hence, a marked term such as $h(x, y) \cdot\{x \mapsto a, z \mapsto b\}$ is considered to be equivalent to $h(x, y) \cdot\{x \mapsto a\}$, since $z$ is not part of the skeleton $h(x, y)$ and, hence, the substitution $\{z \mapsto b\}$ does not identify any marked subterm. Therefore, we define the equivalence on marked terms as the reflexive, symmetric and transitive closure of the relation $\equiv$ including:

1. $s \cdot(\gamma \cup\{x \mapsto t\}) \equiv s \cdot \gamma \quad$ if $\quad x \notin \operatorname{Var}(s) \cup \operatorname{Dom}(\gamma)$.

2. $s \cdot(\gamma \cup\{x \mapsto t\}) \equiv s\{x \mapsto y\} \cdot(\gamma \cup\{y \mapsto t\}) \quad$ if $\quad x \notin \operatorname{Dom}(\gamma)$ and $y \notin \operatorname{Var}(s) \cup \operatorname{Dom}(\gamma)$.

For example, $f(x) \cdot\{x \mapsto a, y \mapsto b\} \equiv f(x) \cdot\{x \mapsto a\} \equiv f(y) \cdot\{y \mapsto a\}$, but $f(x, x) \cdot\{x \mapsto$ $a\} \not \equiv f(x, y) \cdot\{x \mapsto a, y \mapsto a\}$.

For simplicity reasons, in what follows, when considering any set of terms (equations, or clauses) we will assume that the set of marking variables is disjoint from the set of nonmarking variables.

Given a marked term $s \cdot \gamma$ and a substitution $\sigma$ (such that the variables in Dom $(\gamma)$ occur neither in $\operatorname{Dom}(\sigma)$ nor in $\operatorname{Ran}(\sigma))$, we define $(s \cdot \gamma) \sigma=\left.s \sigma \cdot(\gamma \circ \sigma)\right|_{\operatorname{Dom}(\gamma)}$.

For example,

$$
(f(x, y) \cdot\{y \mapsto g(x)\})\{x \mapsto h(x)\}=f(h(x), y) \cdot\{y \mapsto g(h(x))\},
$$

i.e.,

$$
f\left(x, g(x)^{y}\right)\{x \mapsto h(x)\}=f\left(h(x), g(h(x))^{y}\right) .
$$

A marked term $s \cdot \gamma$ is said to be ground if $s \gamma$ is a ground term. If $(s \cdot \gamma) \sigma$ is a ground marked term, then $\sigma$ is called a ground substitution for $s \cdot \gamma$, and $(s \cdot \gamma) \sigma$ is called a ground instance of $s \cdot \gamma$.

The concept of unifier between terms is extended to marked terms as follows: a unifier of two marked terms $s \cdot \gamma$ and $t \cdot \delta$ is a substitution $\sigma$ such that $(s \cdot \gamma) \sigma \equiv(t \cdot \delta) \sigma$. There exists a unique (up to renaming of variables) most general unifier between marked terms, which can be obtained by straightforwardly adapting any unification algorithm for terms.

The usual concepts of (and notation for) position and subterm are extended to marked terms as follows. Let $s \cdot \gamma$ be a marked term. If $p$ is a position of $s$ then $\left.s \cdot \gamma\right|_{p}$ stands for $\left.s\right|_{p} \cdot \gamma$. If $p$ is a position of $s$ such that $\left.s\right|_{p}$ is a variable $x$ in $\operatorname{Dom}(\gamma)$ and $p \cdot q$ is a position of $s \gamma$, with $q \neq \lambda$, then $\left.s \cdot \gamma\right|_{p \cdot q}$ stands for $\left.x \gamma\right|_{q}$. For example,

$$
\begin{gathered}
\left.f\left(x, g(x)^{y}\right)\right|_{2}=g(x)^{y}, \text { and } \\
\left.f\left(x, g(x)^{y}\right)\right|_{2 \cdot 1}=x .
\end{gathered}
$$

We write $s \cdot \gamma \unrhd t \cdot \delta$ if $t \cdot \delta$ occurs in $s \cdot \gamma$, i.e., $\left.s \cdot \gamma\right|_{p} \equiv t \cdot \delta$ for some position $p$, and we write $s \cdot \gamma \triangleright t \cdot \delta$ if $\left.s \cdot \gamma\right|_{p} \equiv t \cdot \delta$ for some position $p \neq \lambda$. Occasionally, we will write $s$ for $s \cdot \gamma$ when $\gamma$ is empty. 
3.2 Orderings on Marked Terms

Let $\succ_{r}$ be a reduction ordering and $R$ be a terminating ground TRS. Moreover, let $\succ$ be a west ordering including $\succ_{r}$ (recall that every reduction ordering can be extended to a west ordering). With these ingredients, we define an ordering $\succ_{R}$ which combines $\succ$ and $\stackrel{+}{\rightarrow}_{R}$. This is the main ordering we will use in our proofs. As we will see, this ordering will make our calculus compatible with demodulation w.r.t. $\succ_{r}$ at the skeletons.

Definition $1\left(\succ_{R}\right)$ Let $\succ$ be a west ordering, $R$ be a terminating ground TRS, and $s \cdot \gamma$ and $t \cdot \delta$ be two ground marked terms. Then $s \cdot \gamma \succ_{R} t \cdot \delta$ iff

(i) $s \succ \cup>t$ or

(ii) $s \doteq t$ and $s \gamma \stackrel{+}{\rightarrow}_{R} t \delta$.

For instance, if $f(x) \succ g(x)$ for all $x$ and $g(a) \rightarrow g(b) \in R$, then $h(f(a)) \succ_{R} f(a) \succ_{R}$ $f\left(a^{x}\right) \succ_{R} g\left(a^{x}\right) \succ_{R} g\left(b^{x}\right) \succ_{R} c^{x}$, since $h(f(a)) \triangleright f(a)>f(x) \succ g(x) \triangleright x$ (recall that $\triangleright$ is included in every west ordering $\succ$ ). Notice that $g\left(a^{x}\right) \succ_{R} g\left(b^{x}\right)$ since $g(x) \doteq g(x)$ and $g(a) \rightarrow_{R} g(b)$.

In what follows, we will consider $\succ_{R}$ as the transitive closure of the relation defined above (composed with the equivalence relation $\equiv$ on marked terms).

Lemma $2 \succ_{R}$ is well-founded.

Proof We proceed by contradiction. First of all we show that the relation $\succ$ quasi-commutes over $>$, i.e., $\gg \circ \succ$ is contained in $\succ 0 \geq$ : if $s_{1} \gg s_{2} \succ s_{3}$ then $s_{1}$ is of the form $s_{2} \sigma$ for some substitution $\sigma$ and, by stability under substitutions of $\succ$, we have that $s_{2} \sigma \succ s_{3} \sigma \geq s_{3}$, i.e., $s_{1} \succ s_{3} \sigma \geq s_{3}$. From this fact and from well-foundedness of $\succ$ and $>$ it follows that $\succ \cup>$ is well-founded (see, e.g., Dershowitz and Jouannaud (1990)). We also have that $(\succ \cup \gg) \circ \doteq$ is well-founded, since $\doteq$ is a congruence and $\succ \cup>$ commutes over $\doteq$. Hence, in a sequence $s_{1} \cdot \gamma_{1} \succ_{R} s_{2} \cdot \gamma_{2} \succ_{R} \cdots$ there can only be finitely many steps by case (i), i.e., from some point on all steps must be by case (ii).

Finally, let $s_{1} \cdot \gamma_{1} \succ_{R} s_{2} \cdot \gamma_{2} \succ_{R} \cdots$ be an infinite sequence with only steps by case (ii), where $s_{1} \gamma_{1}$ is minimal w.r.t. $\stackrel{+}{\rightarrow}_{R}$ (such minimal term must exist since $R$ is terminating). Then since $s_{1} \gamma_{1} \rightarrow_{R} s_{2} \gamma_{2}$ and $s_{2} \cdot \gamma_{2}$ starts an infinite sequence, $s_{2} \gamma_{2}$ contradicts the minimality of $s_{1} \gamma_{1}$.

Another ordering $>_{m}$ which compares the level of the marked positions of (possibly non-ground) marked terms is needed. This ordering is contained in $\succ_{R}$ for ground marked terms.

Definition $2\left(>_{m}\right)$ Let $s \cdot \gamma$ and $t \cdot \delta$ be two marked tems. Then $s \cdot \gamma>_{m} t \cdot \delta$ iff, for every ground substitution $\sigma$ for $s \cdot \gamma$ and $t \cdot \delta$, we have $s \sigma>t \sigma$.

Note that given a substitution $\sigma$ for two marked terms $s \cdot \gamma$ and $t \cdot \delta$, we have $\operatorname{Dom}(\sigma) \cap$ $(\operatorname{Dom}(\gamma) \cup \operatorname{Dom}(\delta))=\emptyset$ by definition. Therefore, since we are not instantiating any marking variable when requiring $s \sigma>t \sigma,>_{m}$ corresponds to the strict subsumption relation $>$ on the skeletons when dealing with ground marked terms. In fact $s \cdot \gamma>_{m} t \cdot \delta$ always implies $s>t$. But, for non-ground marked terms, $s \gg t$ does not necessarily imply $s \cdot \gamma>_{m} t \cdot \delta$. For example, assuming that $x, y, z$ are variables and $a, b, c$ are not:

- $f(a, a) \cdot \emptyset>_{m} f(x, x) \cdot\{x \mapsto a\}$ since $f(a, a) \sigma=f(a, a)>f(x, x)>f(x, x) \sigma$ for every (ground) substitution $\sigma$ such that $x \notin \operatorname{Dom}(\sigma)$. 
- $f(g(x), y) \cdot\{x \mapsto a\}>_{m} f(z, y) \cdot\{z \mapsto b\}$ since $f(g(x), y) \sigma \gg f(z, y) \sigma$ for every ground substitution $\sigma$ such that $\operatorname{Dom}(\sigma) \cap\{x, z\}=\emptyset$.

- $f(g(x), c) \cdot\{x \mapsto a\} \ngtr_{m} f(z, y) \cdot\{z \mapsto b\}$ since, although $f(g(x), c)>f(z, y)$, taking for instance $\sigma=\{y \mapsto a\}$ we have $f(g(x), c) \sigma=f(g(x), c) \ngtr f(z, a)=f(z, y) \sigma$.

We also define the following equivalence relation.

Definition $3\left(\dot{\bar{~}}_{m}\right)$ Let $s \cdot \gamma$ and $t \cdot \delta$ be two marked tems. Then $s \cdot \gamma \dot{=}_{m} t \cdot \delta$ iff, for every ground substitution $\sigma$ for $s \cdot \gamma$ and $t \cdot \delta$, we have $s \sigma \doteq t \sigma$.

Finally, we define $\geq_{m}=>_{m} \cup \doteq_{m}$. We have the following restricted form of monotonicity for $>_{m}$.

Lemma 3 Let $s \cdot \gamma$ and $t \cdot \delta$ be two marked terms such that $\operatorname{Dom}(\gamma) \cap \operatorname{Dom}(\delta)=\emptyset$. If, for some position $p$ of $s$, we have $\left.s\right|_{p} \cdot \gamma>_{m} t \cdot \delta$, then $s \cdot \gamma>_{m} s[t]_{p} \cdot(\gamma \cup \delta)$.

Proof We proceed by contradiction. By definition of $>_{m}$ we have that $\left.s\right|_{p} \sigma>t \sigma$ for every ground substitution $\sigma$ for $\left.s\right|_{p} \cdot \gamma$ and $t \cdot \delta$. Now assume that $s \sigma^{\prime} \ngtr s[t]_{p} \sigma^{\prime}$ for some ground substitution $\sigma^{\prime}$ for $s \cdot \gamma$ and $s[t]_{p} \cdot(\gamma \cup \delta)$. Since $s[t]_{p} \sigma^{\prime}=s \sigma^{\prime}\left[t \sigma^{\prime}\right]_{p}$, we have $s \sigma^{\prime} \ngtr s \sigma^{\prime}\left[t \sigma^{\prime}\right]_{p}$. Now observe that given two terms $t_{1}$ and $t_{2}$ such that $t_{1}>t_{2}$, we have $C\left[t_{1}\right]>C\left[t_{2}\right]$ for every context $C[]$ not sharing any variable with the term $t_{2}$. Therefore, if $s \sigma^{\prime} \ngtr s \sigma^{\prime}\left[t \sigma^{\prime}\right]_{p}$ there must necessarily be some variable $x$ occurring both in $s \sigma^{\prime}[]_{p}$ and in $t \sigma^{\prime}$. But, since $\sigma^{\prime}$ is ground, $x$ must be a marking variable, contradicting $\operatorname{Dom}(\gamma) \cap \operatorname{Dom}(\delta)=\emptyset$.

Since marking variables are never instantiated by substitutions, stability of $>_{m}$ easily follows.

Lemma $4 \rightarrow_{m}$ is stable under substitutions.

Proof Assume that $s \cdot \gamma>_{m} t \cdot \delta$ and $(s \cdot \gamma) \alpha \ngtr_{m}(t \cdot \delta) \alpha$ for some substitution $\alpha$. Then we have $s \alpha \sigma \ngtr t \alpha \sigma$ for some ground substitution $\sigma$ for $(s \cdot \gamma) \alpha$ and $(t \cdot \delta) \alpha$. Now, taking $\beta=$ $\alpha \circ \sigma$, we have that $\beta$ is a ground substitution for $s \cdot \gamma$ and $t \cdot \delta$, and $s \beta \ngtr t \beta$, contradicting $s \cdot \gamma>_{m} t \cdot \delta$.

The same results on monotonicity and stability apply to the relation $\dot{=}_{m}$. Moreover, compatibility of $>_{m}$ with $\doteq_{m}$ follows trivially from compatibility of $>$ with $\doteq$.

\section{Paramodulation with Equations}

In this section we present an ordered paramodulation calculus for sets of equations. In the following, we assume that $\succ_{r}$ is a given reduction ordering and $\succ$ is a west ordering including $\succ_{r}$.

In the following we will assume that the marked terms of every equation do share the same substitution (if necessary, the substitutions can be extended). Moreover, variables (both marking and non-marking) of each pair of equations will be considered to be disjoint (if necessary, the variables can be renamed). 
4.1 The Inference System

Definition $4(\mathscr{E})$ Let $E$ be a set of marked equations. The inference system $\mathscr{E}$ for $E$ consists of the following single inference rule:

Paramodulation:

$$
\frac{l \cdot \delta \simeq r \cdot \delta \quad s \cdot \gamma \simeq t \cdot \gamma}{\left(s^{\prime} \cdot \gamma^{\prime} \simeq t \cdot \gamma\right) \sigma} \quad \text { if }
$$

1. $\sigma=m g u\left(l \delta,\left.s \gamma\right|_{p}\right)$, the most general unifier of $l \delta$ and $\left.s \gamma\right|_{p}$ for some position $p$ of $s \gamma$,

2. $\left.s \gamma\right|_{p}$ is not a variable,

3. for some ground substitution $\theta$, we have $l \delta \sigma \theta \succ r \delta \sigma \theta$ and, if $p=\lambda$, then we also have $s \gamma \sigma \theta \succ t \gamma \sigma \theta$, and

4. (a) $s^{\prime} \cdot \gamma^{\prime}=s[r \delta]_{p} \cdot \gamma$ if $p$ is a non-marked position of $s$ and $s \sigma \succ s[r \delta]_{p} \sigma$,

(b) $s^{\prime} \cdot \gamma^{\prime}=s[r]_{p} \cdot(\gamma \cup \delta)$ if $p$ is a non-marked position of $s, s \sigma \nsucc s[r \delta]_{p} \sigma$ and $\left(\left.s\right|_{p}\right.$. $\gamma) \sigma>_{m}(r \cdot \delta) \sigma$

(c) $s^{\prime} \cdot \gamma^{\prime}=s[x]_{p} \cdot(\gamma \cup\{x \mapsto r \delta\})$, where $x$ is a fresh variable, if $p$ is a non-marked position of $s, s \sigma \nsucc s[r \delta]_{p} \sigma$ and $\left(\left.s\right|_{p} \cdot \gamma\right) \sigma \ngtr_{m}(r \cdot \delta) \sigma$, and

(d) $s^{\prime} \cdot \gamma^{\prime}=s[y]_{q} \cdot\left(\gamma \cup\left\{y \mapsto x \gamma[r \delta]_{q^{\prime}}\right\}\right)$, where $y$ is a fresh variable, if $p=q \cdot q^{\prime}$ and $\left.s\right|_{q}$ is a variable $x$ in $\operatorname{Dom}(\gamma)$, i.e., $p$ is below a marked position $q$ of $s \cdot \gamma$.

Note that since the marking variables introduced by the previous inference rule are fresh, we are mantaining the invariant on disjointness of marking and non-marking variables. The following simple example illustrates basic applications of this paramodulation rule.

Example 2 Let $\succ_{r}$ be a reduction including $f(a) \succ_{r} f(b)$ and $g(b) \succ_{r} g(a)$. Then necessarily $a \nsucc_{r} b$ and $b \nsucc_{r} a$. Let $E$ denote the following set of equations:
1) $a \simeq b$
2) $f(f(a)) \simeq c$
3) $g(a) \simeq c$

Let $\succ$ be a west ordering which is an extension of $\succ_{r}$, including $a \succ b$. Then, since $a \succ b$ and $f(a) \succ_{r} f(b)$, we have $\left.f(f(a))\right) \succ f(f(b))$, and hence the inference by paramodulation with 1 into 2 applies case $4 \mathrm{a}$ and gives us

$$
\text { 4) } f(f(b)) \simeq c
$$

Instead, since $a \succ b$ but $g(a) \nsucc g(b)$ and $a \ngtr_{m} b$, the inference by paramodulation with 1 into 3 applies case $4 \mathrm{c}$ and gives us

$$
\text { 5) } g\left(b^{x}\right) \simeq c
$$

Conditions 1 to 3 are the usual restrictions of the ordered paramodulation inference rule, after forgetting the marks of the terms. Here follows some more examples illustrating conditions 4a-4d.

Assume that $g(f(x)) \succ_{r} f(a)$. Then we have the following inference applying case 4a:

$$
\frac{g(y) \cdot\{y \mapsto f(x)\} \simeq y \cdot\{y \mapsto f(x)\} \quad h(g(f(z))) \cdot\{z \mapsto a\} \simeq t}{h(f(a))=t}
$$


i.e.,

$$
\frac{g\left(f(x)^{y}\right) \simeq f(x)^{y} \quad h\left(g\left(f\left(a^{z}\right)\right)\right) \simeq t}{h(f(a))=t}
$$

with $\sigma=\operatorname{mgu}(g(f(x)), g(f(a)))=\{x \mapsto a\}$ (condition 1), since $g(f(a))$ is not a variable (condition 2), $g(f(a)) \succ f(a)$ (condition 3) and $h(g(f(z))) \sigma=h(g(f(z))) \succ h(f(a))=$ $h(f(x)) \sigma$ (condition 4a) thanks to monotonicity of $\succ_{r}$ and inclusion of $\succ_{r}$ in $\succ$. Notice that we are forgetting the marks in order to perform unification. Moreover, instead of introducing the marked term $f(a)^{y}$ in the conclusion (i.e., the right hand side of the left premise with the unifier applied) we can indeed forget the mark and introduce $f(a)$ instead, if there is a decrease w.r.t. $\succ$ when considering the marks of the rightmost premise as variables. Observe that, as a particular case, if both premises have no marks and the leftmost premise can be oriented with respect to the reduction ordering at hand, then condition $4 \mathrm{a}$ is always fulfilled, and hence no marks are introduced in the conclusion, making our inference rule coincide with the usual ordered paramodulation inference rule.

If we have $g(f(a)) \succ g(a)$ but $h(g(f(a))) \nsucc h(g(a))$, which is possible since $\succ$ does not need to be monotonic, then we have the following inference applying case $4 \mathrm{~b}$ :

$$
\frac{g\left(f(x)^{y}\right) \simeq g\left(x^{z}\right) \quad h(g(f(a))) \simeq t}{h\left(g\left(a^{z}\right)\right)=t}
$$

with $\sigma=\{x \mapsto a\}$. Observe that, although $h(g(f(a))) \nsucc h(g(a))$, we have $g(f(a))>_{m} g\left(a^{z}\right)$ since $g(z)$ subsumes $g(f(a))$. What we are obtaining here is a term with marks at a higher position.

Following the previous example, if $g(f(a)) \succ g(a)$ but $h(g(f(a))) \nsucc h(g(a))$ then we have the following inference applying case $4 \mathrm{c}$ :

$$
\frac{g\left(f(x)^{y}\right) \simeq g(x) \quad h(g(f(a))) \simeq t}{h\left(g(a)^{z}\right)=t}
$$

with $\sigma=\{x \mapsto a\}$. Notice that in this case we have $g(f(a)) \ngtr_{m} g(a)$. By adding a mark at the inference position we guarantee a decreasing w.r.t. $>_{m}$. But, in fact, it would suffice to add any marking variables (possibly at lower positions) guaranteeing a decrease w.r.t. $>_{m}$ (e.g., on top of $a$ ).

Finally, case $4 \mathrm{~d}$ amounts to inferences which take place below marks. For example, if $g(f(a)) \succ f(a)$, then we have the following inference applying case $4 \mathrm{~d}$ :

$$
\frac{g\left(f(x)^{y}\right) \simeq f(x)^{y} \quad h(g(f(a)))^{z} \simeq t}{h(f(a))^{z}=t}
$$

In this case, no additional mark is needed in the conclusion of the inference.

As said in the introduction, this inference system is roughly the same as in Bofill et al (2003), with the main difference that some subterms become marked in the equations. But these marks only have effect for redundancy purposes. So the amount of inferences by paramodulation here is the same as in Bofill et al (2003). The basic idea is to mark out problematic (w.r.t. redundancy notions) subterms. Marked subterms will be seen as variables for redundancy purposes, as redundancy will be defined w.r.t. $\succ_{R}$ (see Definition 1). Hence, the more marks we have to introduce, the less redundancy we have. Marked subterms will roughly be those terms occurring as small sides (w.r.t. $\succ$ ) of equations that cannot be oriented by the reduction ordering $\succ_{r}$ included in $\succ$. The intuition is that rewriting with 
those unorientable equations w.r.t. $\succ_{r}$ may interfere with simplification by rewriting with the orientable ones, and possibly lead to incompleteness of the calculus (see, e.g., Example 6 of Section 5.5). It is worth noting that, whereas we do not require totality of $\succ_{r}$, if all equations at hand can be handled by $\succ_{r}$ then no (new) marks will be introduced in the conclusion of any inference (notice that in this situation case $4 \mathrm{a}$ of the paramodulation inference rule always applies).

\subsection{Redundancy Notions: a Static View}

Here we introduce redundancy notions from a static point of view, that is, by first defining the notion of saturated set, regardless of how this saturated set can be obtained. For this, we define some abstract redundancy notions for inferences. The problem of how to compute such saturated sets is addressed in Section 5.4, while several practical redundancy notions, fitting in the abstract notions defined so far, are discussed afterwards, in Section 5.5.

In order to define the redundancy notions and later on prove the refutation completeness of our calculus, we have first to introduce the notion of irreducible instances. This is used here to avoid the use of the Lifting lemma, since in our case inferences below variables cannot be guaranteed to decrease.

Definition 5 (Variable Irreducibility) Let $R$ be a TRS, $(t \cdot \gamma) \sigma$ an instance of a marked term $t \cdot \gamma$, and $x$ a variable in $\operatorname{Var}(t \gamma)$. Then $x$ is said to be variable irreducible in $(t \cdot \gamma) \sigma$ w.r.t. $R$ if $x \sigma$ is irreducible w.r.t. $R$. Moreover, $(t \cdot \gamma) \sigma$ is said to be variable irreducible w.r.t. $R$ if all variables in $\operatorname{Var}(t \gamma)$ are variable irreducible in $(t \cdot \gamma) \sigma$ w.r.t. $R$.

Definition $6\left(\operatorname{irred}_{R}(E)\right)$ Let $E$ be a set of marked equations and $R$ be a TRS. By $\operatorname{irred}_{R}(E)$ we denote the set of all variable irreducible ground instances of equations in $E$ w.r.t. $R$.

Definition $7\left(\operatorname{irred}_{R}(\pi)\right)$ Let $\pi$ be an inference with premises $e_{1}, e_{2}$ and conclusion $d$ and $R$ be a TRS. By $\operatorname{irred}_{R}(\pi)$ we denote the set all ground instances $\pi \sigma$ of $\pi$ s.t. $e_{1} \sigma$ and $e_{2} \sigma$ are variable irreducible w.r.t. $R$,

Let $>$ be a well-founded ordering on marked equations. By $E^{<e}$ we denote the set of all equations $d$ in $E$ such that $e>d$. An analogous notation is used for quasi-orderings $\geq$. We define $\succeq_{R}$ as $\succ_{R} \cup \equiv$, where $\equiv$ denotes the equivalence of marked terms as defined in Section 3 .

Definition 8 (Redundancy of Inferences) Let $E$ be a set of marked equations and $R$ a terminating ground TRS. A ground inference by $\mathscr{E}$ with premises $e_{1}, e_{2}$ and conclusion $d$ is redundant in $E$ w.r.t. $R$ if we have

$$
\begin{aligned}
& R^{*} \cup \text { Forget }_{\left(\operatorname{irred}_{R}(E)^{\prec_{R}^{m u l} e_{2}}\right)} \\
& \left.\quad \cup \text { Forget } \operatorname{irred}_{R}(E)^{\preceq_{R}^{m u l} d}\right) \models \operatorname{Forget}(d) .
\end{aligned}
$$

An inference $\pi$ by $\mathscr{E}$ is redundant in $E$ if for every terminating ground TRS $R$ we have that all inferences in $\operatorname{irred}_{R}(\pi)$ are redundant in $E$ w.r.t. $R$.

Notice that universally quantifying the TRS $R$ allows us to capture the particular TRS $R_{E}$ (see Definition 11) defining the model, which cannot be known in advance.

Redundant inferences are unnecessary and, therefore, we are interested in computing the closure of a set of equations with respect to $\mathscr{E}$ up to redundancy: 
Definition 9 (Saturatedness) A set $E$ of marked equations is saturated with respect to $\mathscr{E}$ if every inference by $\mathscr{E}$ with premises in $E$ is redundant in $E$.

\subsection{Model Generation}

Definition $10(\operatorname{gnd}(E))$ Let $E$ be a set of (marked) equations. By $\operatorname{gnd}(E)$ we denote the set of all ground instances of equations in $E$.

Here we show how to build an equational model of Forget $(E)$ for a given set of marked equations $E$. To this end, we define a ground term rewrite system $R_{E}$ from Forget $(g n d(E))$, by induction on $\succ^{m u l}$. This TRS coincides with the one generated in Bofill et al (2003) for the equational case, where no marks are used. As we show in Theorem $1, R_{E}^{*}$ will be a model of $\operatorname{Forget}(E)$ whenever $E$ is saturated with respect to $\mathscr{E}$.

In the following, we assume $E$ being a set of equations such that Forget $(E)$ does not contain any equation of the form $x \simeq t$ where $x$ is a variable not occurring in $t$ since, otherwise, the theory collapses.

Definition $11\left(R_{E}\right)$ Let $e$ be an equation of the form $l \simeq r$ in Forget $(\operatorname{gnd}(E))$. Then $e$ generates the rule $l \rightarrow r$ in $R_{E}$ if

1. $l \succ r$,

2. $l$ is irreducible by $R_{E}^{e}$, and

3. $r$ is irreducible by $R_{E}^{e}$ at non-topmost positions,

where $R_{E}^{e}$ denotes the set of rules generated by all equations $d$ in $\operatorname{Forget}(\operatorname{gnd}(E))$ such that $e \succ^{m u l} d$. We denote by $R_{E}$ the set of rules generated by all equations in Forget $(\operatorname{gnd}(E))$.

Property 1 (Bofill et al (2003)) Let $E$ be a set of (marked) equations. Then for all rules $l \rightarrow r$ in $R_{E}$ we have that

1. $l$ is irreducible by $R_{E} \backslash\{l \rightarrow r\}$, and

2. $r$ is irreducible by $R_{E}$ at non-topmost positions.

Lemma 5 (Bofill et al (2003)) For every set of (marked) equations $E, R_{E}$ is convergent.

Lemma 6 Let $E$ be a set of marked equations $E$. Then for all rules $l \rightarrow r$ in $R_{E}$ we have that

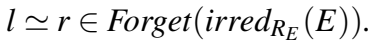

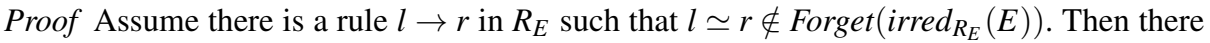
is an equation $l^{\prime} \simeq r^{\prime}$ in Forget $(E)$ such that $l^{\prime} \sigma \equiv l$ and $r^{\prime} \sigma \equiv r$ and $x \sigma$ is reducible by $R_{E}$ for some variable $x$ occurring in $l^{\prime}$ or $r^{\prime}$. Now observe that, by Property $1, x$ can only occur either at topmost position of $l^{\prime}$ or of $r^{\prime}$. But, since we are assuming that Forget $(E)$ does not contain equations of the form $x \simeq t$ where $x$ does not occur in $t$, then $l^{\prime} \simeq r^{\prime}$ must be of the form $x \simeq x$, contradicting $l \succ r$.

Now we prove that, if $E$ is saturated with respect to $\mathscr{E}$, then $R_{E}^{*}$ is a model of Forget $(E)$. We begin by showing that we have an equational model of all variable irreducible instances of $E$ w.r.t. $R_{E}$, i.e., that $R_{E}^{*}=$ Forget $\left(\operatorname{irred}_{R_{E}}(E)\right)$.

Lemma 7 Let $E$ be a set of marked equations. If $E$ is saturated with respect to $\mathscr{E}$, then $R_{E}^{*}=\operatorname{Forget}\left(\operatorname{irred}_{R_{E}}(E)\right)$. 
Proof We proceed by induction w.r.t. $\succ_{R}^{m u l}$ taking $R=R_{E}$ (see Definition 1). A contradiction is derived from the existence of a minimal w.r.t. $\succ_{R}^{m u l}$ ground instance $e$ in $\operatorname{irred}_{R_{E}}(E)$ of the form $(s \cdot \gamma) \theta \simeq(t \cdot \gamma) \theta$ of an equation $s \cdot \gamma \simeq t \cdot \gamma$ in $E$, such that $R_{E}^{*} \not \neq \operatorname{Forget}(e)$.

Since $R_{E}^{*} \not \models$ Forget $(e)$, then Forget $(e)$ has not generated any rule of $R_{E}$ and, moreover, we necessarily have $s \gamma \theta \not \equiv t \gamma \theta$. We will assume, w.l.o.g., that $s \gamma \theta \succ t \gamma \theta$. Now, since Forget $(e)$ has not generated any rule of $R_{E}$, it must be either because $s \gamma \theta$ is reducible by some rule in $R_{E}$, or $t \gamma \theta$ is reducible by some rule in $R_{E}$ at some non-topmost position. We consider the case where $s \gamma \theta$ is reducible (the other one is analogous). Then there is an equation $l \cdot \delta \simeq r \cdot \delta$ in $E$ that has generated a rule $l \delta \theta \rightarrow r \delta \theta$ in $R_{E}$ (we can use the same $\theta$ since the equations do not share variables), such that $\left.s \gamma \theta\right|_{p} \equiv l \delta \theta$ for some position $p$. Moreover, $p$ must be a non-variable position of $s \gamma$, as $e$ is variable irreducible w.r.t. $R_{E}$. Then there is an inference by paramodulation,

$$
\frac{l \cdot \delta \simeq r \cdot \delta \quad s \cdot \gamma \simeq t \cdot \gamma}{\left(s^{\prime} \cdot \gamma^{\prime} \simeq t \cdot \gamma\right) \sigma}
$$

where $\sigma=m g u\left(l \delta,\left.s \gamma\right|_{p}\right)$, and

1. $s^{\prime} \cdot \gamma^{\prime}=s[r \delta]_{p} \cdot \gamma$ if $p$ is a non-marked position of $s$ and $s \sigma \succ s[r \delta]_{p} \sigma$,

2. $s^{\prime} \cdot \gamma^{\prime}=s[r]_{p} \cdot(\gamma \cup \delta)$ if $p$ is a non-marked position of $s, s \sigma \nsucc s[r \delta]_{p} \sigma$ and $\left(\left.s\right|_{p} \cdot \gamma\right) \sigma>_{m}(r$. $\delta) \sigma$,

3. $s^{\prime} \cdot \gamma^{\prime}=s[x]_{p} \cdot(\gamma \cup\{x \mapsto r \delta\})$, where $x$ is a fresh variable, if $p$ is a non-marked position of $s, s \sigma \nsucc s[r \delta]_{p} \sigma$ and $\left(\left.s\right|_{p} \cdot \gamma\right) \sigma \ngtr_{m}(r \cdot \delta) \sigma$, and

4. $s^{\prime} \cdot \gamma^{\prime}=s[y]_{q} \cdot\left(\gamma \cup\left\{y \mapsto x \gamma[r \delta]_{q^{\prime}}\right\}\right)$, where $y$ is a fresh variable, if $p=q \cdot q^{\prime}$ and $\left.s\right|_{q}$ is a variable $x$ in $\operatorname{Dom}(\gamma)$, i.e., $p$ is below a marked position $q$ of $s \cdot \gamma$.

Therefore, the conclusion of the previous inference has an instance $d$ of the form $\left(s^{\prime} \cdot \gamma^{\prime} \simeq\right.$ $t \cdot \gamma) \theta$, and Forget $(d)$ corresponds to $\left(s \gamma[r \delta]_{p} \simeq t \gamma\right) \theta$. Moreover, $d$ is variable irreducible w.r.t. $R_{E}$, since $e$ is variable irreducible by assumption and $l \delta \theta \simeq r \delta \theta$ is variable irreducible by Lemma 6. Now we show that $e \succ_{R}^{m u l} d$ (with $R=R_{E}$ ):

- If $s^{\prime} \cdot \gamma^{\prime}$ is as indicated in case 1 then, since $\succ$ is stable under substitutions, we have that $s \theta \succ s[r \delta]_{p} \theta$ and hence $(s \cdot \gamma) \theta \succ_{R}\left(s^{\prime} \cdot \gamma^{\prime}\right) \theta$ by case (i) of definition of $\succ_{R}$.

- If $s^{\prime} \cdot \gamma^{\prime}$ is as indicated in case 2 then, by monotonicity and stability of $>_{m}$ (Lemmas 3 and 4), we have $(s \cdot \gamma) \theta>_{m}\left(s[r]_{p} \cdot(\gamma \cup \delta)\right) \theta$, i.e., $(s \cdot \gamma) \theta>_{m}\left(s^{\prime} \cdot \gamma^{\prime}\right) \boldsymbol{\theta}$. Therefore $s \boldsymbol{\theta}>s^{\prime} \theta$ and hence $(s \cdot \gamma) \theta \succ_{R}\left(s^{\prime} \cdot \gamma^{\prime}\right) \theta$ by case (i) of definition of $\succ_{R}$ as before.

- If $s^{\prime} \cdot \gamma^{\prime}$ is as indicated in case 3 then $s \cdot \gamma>_{m} s^{\prime} \cdot \gamma^{\prime}$ and, by stability of $>_{m},(s \cdot \gamma) \theta>_{m}\left(s^{\prime}\right.$. $\left.\gamma^{\prime}\right) \theta$, which lets us conclude that $(s \cdot \gamma) \theta \succ_{R}\left(s^{\prime} \cdot \gamma^{\prime}\right) \theta$ by case (i) of definition of $\succ_{R}$ as in the previous case.

- Finally, if $s^{\prime} \cdot \gamma^{\prime}$ is as indicated in case 4 , then $s \cdot \gamma \geq_{m} s^{\prime} \cdot \gamma^{\prime}$ and moreover $(s \cdot \gamma) \theta \geq_{m}\left(s^{\prime}\right.$. $\left.\gamma^{\prime}\right) \theta$, which implies $s \theta \geq s^{\prime} \theta$. Additionally, we have that $s \gamma \theta \rightarrow_{R_{E}} s \gamma[r \delta]_{p} \theta$. Therefore $(s \cdot \gamma) \theta \succ_{R}\left(s^{\prime} \cdot \gamma^{\prime}\right) \theta$ by zero or one step of case (i) of definition of $\succ_{R}$, followed by one step of case (ii) of definition of $\succ_{R}$ (with $R=R_{E}$ ).

Now, since $E$ is saturated w.r.t. $\mathscr{E}$, this inference is redundant in $E$. Therefore we have

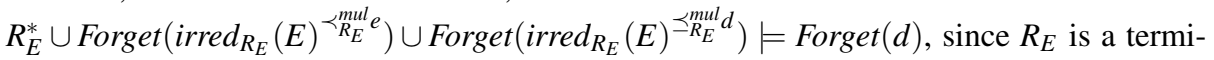
nating ground TRS and both $e$ and $d$ are variable irreducible w.r.t. $R_{E}$. On the other hand, we have $e \succ_{R_{E}}^{m u l} d$ and $R_{E}^{*}=$ Forget $\left(\right.$ irred $_{R_{E}}(E)^{\prec_{R_{E}}^{m u l} e}$ ) by minimality of $e$, and hence $R_{E}^{*} \models$ $\left.\operatorname{Forget}_{\left(\operatorname{irred}_{R_{E}}(E)^{\mathfrak{R}_{E} m u l} d\right.}\right)$. Altogether, this gives us $R_{E}^{*}=\operatorname{Forget}(d)$ and, since $R_{E}^{*}=l \delta \theta \simeq$ $r \delta \theta$, it contradicts $R_{E}^{*} \not \models$ Forget $(e)$. 
Theorem 1 Let $E$ be a set of marked equations. If $E$ is saturated with respect to $\mathscr{E}$, then $R_{E}^{*}=\operatorname{Forget}(E)$.

Proof For each ground instance $e \sigma$ of an equation $e$ in $E$ there is an equation $e \sigma^{\prime}$ in irred $_{R_{E}}(E)$ where, for every variable $x$ of $e, x \sigma^{\prime}$ is the normal form of $x \sigma$ with respect to $R_{E}$. Now, if $E$ is saturated with respect to $\mathscr{E}$, we have that $R_{E}^{*} \models$ Forget $\left(e \sigma^{\prime}\right)$ by Lemma 7, and hence $R_{E}^{*}=\operatorname{Forget}(e \sigma)$ as well.

\section{General Clauses}

Here we extend the presented calculus to general first order clauses, and prove it complete. We consider that in each clause with a non-empty antecedent one of its negative equations, the one that is written underlined, has been selected. We use the orderings defined in Section 3.2, and assume that all marked terms in the premises of an inference share a unique substitution.

\subsection{The Inference System}

Definition $12(\mathscr{G})$ Let $S$ be a set of general first order clauses. Our inference system $\mathscr{G}$ consists of the following four inference rules:

\section{Paramodulation right:}

$$
\frac{\rightarrow l \cdot \gamma \simeq r \cdot \gamma, \Delta_{1} \quad \rightarrow s \cdot \gamma \simeq t \cdot \gamma, \Delta_{2}}{\left(\rightarrow s^{\prime} \cdot \gamma^{\prime} \simeq t \cdot \gamma, \Delta_{1}^{\prime}, \Delta_{2}\right) \sigma} \quad \text { if }
$$

1. $\sigma=m g u\left(l \gamma,\left.s \gamma\right|_{p}\right)$ for some position $p$.

2. $\left.s \gamma\right|_{p}$ is not a variable.

3. For some ground substitution $\theta$, we have that $l \gamma \sigma \theta \succ r \gamma \sigma \theta$ and $(l \gamma \simeq r \gamma) \sigma \theta \succ^{m u l}$ Forget $(e) \sigma \theta$ for all equations $e$ in $\Delta_{1}$ and, if $p=\lambda$, then $s \gamma \sigma \theta \succ t \gamma \sigma \theta$ and $(s \gamma \simeq$ $t \gamma) \sigma \theta \succ^{m u l}$ Forget $(e) \sigma \theta$ for all equations $e$ in $\Delta_{2}$.

4. (a) $s^{\prime} \cdot \gamma^{\prime}=s[r \delta]_{p} \cdot \gamma$ if $p$ is a non-marked position of $s$ and $s \sigma \succ s[r \delta]_{p} \sigma$,

(b) $s^{\prime} \cdot \gamma^{\prime}=s[r]_{p} \cdot(\gamma \cup \delta)$ if $p$ is a non-marked position of $s, s \sigma \nsucc s[r \delta]_{p} \sigma$ and $\left(\left.s\right|_{p}\right.$. $\gamma) \sigma>_{m}(r \cdot \delta) \sigma$

(c) $s^{\prime} \cdot \gamma^{\prime}=s[x]_{p} \cdot(\gamma \cup\{x \mapsto r \delta\})$, where $x$ is a fresh variable, if $p$ is a non-marked position of $s, s \sigma \nsucc s[r \delta]_{p} \sigma$ and $\left(\left.s\right|_{p} \cdot \gamma\right) \sigma \ngtr_{m}(r \cdot \delta) \sigma$, and

(d) $s^{\prime} \cdot \gamma^{\prime}=s[y]_{q} \cdot\left(\gamma \cup\left\{y \mapsto x \gamma[r \delta]_{q^{\prime}}\right\}\right)$, where $y$ is a fresh variable, if $p=q \cdot q^{\prime}$ and $\left.s\right|_{q}$ is a variable $x$ in $\operatorname{Dom}(\gamma)$, i.e., $p$ is below a marked position $q$ of $s \cdot \gamma$.

5. For each equation $u \cdot \gamma \simeq v \cdot \gamma$ in $\Delta_{1}$ there is an equation $u^{\prime} \cdot \gamma^{\prime} \simeq v^{\prime} \cdot \gamma^{\prime}$ in $\Delta_{1}^{\prime}$ (and viceversa), where
(a) $u^{\prime} \cdot \gamma^{\prime}=u \gamma$ if $s \sigma \succ u \gamma \sigma$ or $t \sigma \succ u \gamma \sigma,{ }^{4}$
(b) $u^{\prime} \cdot \gamma^{\prime}=u \cdot \gamma$ if the previous case does not apply and $(s \cdot \gamma) \sigma>_{m}(u \cdot \gamma) \sigma$ or $(t$. $\gamma) \sigma>_{m}(u \cdot \gamma) \sigma$, and
(c) $u^{\prime} \cdot \gamma^{\prime}=x \cdot\{x \mapsto u \gamma\}$ for some fresh variable $x$ otherwise, and analogously for $v^{\prime} \cdot \gamma^{\prime}$.

\footnotetext{
4 Recall that $u \gamma$ is the same as $u \gamma \cdot \emptyset$.
} 
Paramodulation left:

$$
\frac{\rightarrow l \cdot \gamma \simeq r \cdot \gamma, \Delta_{1} \quad \Gamma, s \cdot \gamma \simeq t \cdot \gamma \rightarrow \Delta_{2}}{\left(\Gamma, s^{\prime} \cdot \gamma^{\prime} \simeq t \cdot \gamma \rightarrow \Delta_{1}^{\prime}, \Delta_{2}\right) \sigma} \quad \text { if }
$$

1. $\sigma=m g u\left(l \gamma,\left.s \gamma\right|_{p}\right)$ for some position $p$.

2. $\left.s \gamma\right|_{p}$ is not a variable.

3. For some ground substitution $\theta$, we have that $l \gamma \sigma \theta \succ r \gamma \sigma \theta$ and $(l \gamma \simeq r \gamma) \sigma \theta \succ^{m u l}$ Forget $(e) \sigma \theta$ for all equations $e$ in $\Delta_{1}$ and, if $p=\lambda$, then $s \gamma \sigma \theta \succ t \gamma \sigma \theta$.

4. (a) $s^{\prime} \cdot \gamma^{\prime}=s[r \delta]_{p} \cdot \gamma$ if $p$ is a non-marked position of $s$ and $s \sigma \succ s[r \delta]_{p} \sigma$,

(b) $s^{\prime} \cdot \gamma^{\prime}=s[r]_{p} \cdot(\gamma \cup \delta)$ if $p$ is a non-marked position of $s, s \sigma \nsucc s[r \delta]_{p} \sigma$ and $\left(\left.s\right|_{p}\right.$. $\gamma) \sigma>_{m}(r \cdot \delta) \sigma$

(c) $s^{\prime} \cdot \gamma^{\prime}=s[x]_{p} \cdot(\gamma \cup\{x \mapsto r \delta\})$, where $x$ is a fresh variable, if $p$ is a non-marked position of $s, s \sigma \nsucc s[r \delta]_{p} \sigma$ and $\left(\left.s\right|_{p} \cdot \gamma\right) \sigma \ngtr_{m}(r \cdot \delta) \sigma$, and

(d) $s^{\prime} \cdot \gamma^{\prime}=s[y]_{q} \cdot\left(\gamma \cup\left\{y \mapsto x \gamma[r \delta]_{q^{\prime}}\right\}\right)$, where $y$ is a fresh variable, if $p=q \cdot q^{\prime}$ and $\left.s\right|_{q}$ is a variable $x$ in $\operatorname{Dom}(\gamma)$, i.e., $p$ is below a marked position $q$ of $s \cdot \gamma$.

5. For each equation $u \cdot \gamma \simeq v \cdot \gamma$ in $\Delta_{1}$ there is an equation $u^{\prime} \cdot \gamma^{\prime} \simeq v^{\prime} \cdot \gamma^{\prime}$ in $\Delta_{1}^{\prime}$ (and viceversa), where

(a) $u^{\prime} \cdot \gamma^{\prime}=u \gamma$ if $s \sigma \succ u \gamma \sigma$ or $t \sigma \succ u \gamma \sigma$,

(b) $u^{\prime} \cdot \gamma^{\prime}=u \cdot \gamma$ if the previous case does not apply and $(s \cdot \gamma) \sigma>_{m}(u \cdot \gamma) \sigma$ or $(t$. $\gamma) \sigma>_{m}(u \cdot \gamma) \sigma$, and

(c) $u^{\prime} \cdot \gamma^{\prime}=x \cdot\{x \mapsto u \gamma\}$ for some fresh variable $x$ otherwise,

and analogously for $v^{\prime} \cdot \gamma^{\prime}$.

\section{Equality resolution:}

$$
\frac{\Gamma, s \cdot \gamma \simeq t \cdot \gamma \rightarrow \Delta}{(\Gamma \rightarrow \Delta) \sigma} \quad \text { if } \quad \sigma=m g u(s \gamma, t \gamma) .
$$

\section{Equality factoring:}

$$
\frac{\rightarrow l \cdot \gamma \simeq r \cdot \gamma, s \cdot \gamma \simeq t \cdot \gamma, \Delta}{\left(r^{\prime} \cdot \gamma^{\prime} \simeq t \cdot \gamma \rightarrow l \cdot \gamma \simeq r \cdot \gamma, \Delta\right) \sigma} \quad \text { if }
$$

1. $\sigma=m g u(l \gamma, s \gamma)$.

2. For some ground substitution $\theta$, we have that $l \gamma \sigma \theta \succ r \gamma \sigma \theta$ and $(l \gamma \simeq r \gamma) \sigma \theta \succeq^{m u l}$ Forget $(e) \sigma \theta$ for all equations $e$ in $\{s \cdot \gamma \simeq t \cdot \gamma\} \cup \Delta$.

3. (a) $r^{\prime} \cdot \gamma^{\prime}=r \gamma$ if $s \sigma \succ r \gamma \sigma$,

(b) $r^{\prime} \cdot \gamma^{\prime}=r \cdot \gamma$ if the previous case does not apply and $(s \cdot \gamma) \sigma>_{m}(r \cdot \gamma) \sigma$, and

(c) $r^{\prime} \cdot \gamma^{\prime}=x \cdot\{x \mapsto r \gamma\}$ for some fresh variable $x$ otherwise.

The following example illustrates how the previous inference system works.

Example 3 Let $\succ_{r}$ be a reduction ordering including

$$
\begin{gathered}
g(a) \succ_{r} g(f(a)) \succ_{r} h(f(a)) \succ_{r} h(a), \\
h(a) \succ_{r} f(a), \text { and } h(a) \succ_{r} a .
\end{gathered}
$$

Observe that, on the one hand, $f(a)$ and $a$ must be incomparable in $\succ_{r}$ and, on the other hand, we must have $f(a) \succ a$ in any west ordering $\succ$. 
Let $S$ denote the following inconsistent set of general first order clauses:

1) $\rightarrow g(f(a)) \simeq h(a), g(a) \simeq h(f(a))$

2) $g(x) \simeq h(y) \rightarrow x \simeq y$

3) $g(x) \simeq h(x) \rightarrow$

We illustrate how the empty clause can be derived from $S$ with the inference system $\mathscr{G}$. In order to ease the reading, we underline the terms that are unified in an inference.

First of all, an inference by paramodulation left with 1 into 2, i.e., with $\rightarrow g(f(a)) \simeq$ $h(a), g(a) \simeq h(f(a))$ into $g(x) \simeq h(y) \rightarrow x \simeq y$ is possible, giving

$$
\text { 4) } h(f(a)) \simeq h(y) \rightarrow a \simeq y, g(f(a)) \simeq h(a)
$$

Observe that this is a valid inference, with $\sigma=\{x \mapsto a\}$, since $g(a)$ is strictly maximal w.r.t. $\succ$ in the left premise (where $\succ$ is the west ordering including $\succ_{r}$ ), and $g(x) \sigma \theta \succ h(y) \sigma \theta$ for some ground substitution $\theta$ (take, e.g., $\theta=\{y \mapsto a\}$ ). Moreover, no term needs to be marked in the conclusion since, on the one hand, $g(a) \succ h(f(a))$ (i.e., case $4 \mathrm{a}$ applies) and, on the other hand, $g(a) \succ g(f(a))$ and $g(a) \succ h(a)$ (i.e., case 5a applies).

Now, an inference by equality resolution on 4 is possible, giving

$$
\text { 5) } \rightarrow a \simeq f(a), g(f(a)) \simeq h(a)
$$

Then, an inference by paramodulation left with 5 into 2 , i.e., with $\rightarrow a \simeq f(a), g(f(a)) \simeq$ $h(a)$ into $g(x) \simeq h(y) \rightarrow x \simeq y$ is possible, giving

$$
\text { 6) } h(a) \simeq h(y) \rightarrow f(a) \simeq y, a \simeq f(a)
$$

Notice that $g(f(a))$ is maximal w.r.t. $\succ$ in its premise. Moreover again no marks need to be added to the conclusion since, on the one hand, $g(f(a)) \succ h(a)$ (i.e., case $4 \mathrm{a}$ applies) and, on the other hand, $g(a) \succ f(a)$ and $g(a) \succ a$ (i.e., case 5a applies).

Now, an inference by equality resolution on 6 gives us

$$
\text { 7) } f(a) \simeq a, a \simeq f(a)
$$

And with an inference by equality factoring on 7 we obtain

$$
\text { 8) } a \simeq a \rightarrow f(a) \simeq a
$$

Notice that, although $f(a) \nsucc_{r} a$, we have that $f(a) \succ a$ and hence no mark is necessary in the conclusion.

Now, an inference by equality resolution on 8 gives us

$$
\text { 9) } \rightarrow f(a) \simeq a
$$

Then there is an inference by paramodulation right with 9 into 1 , that is with $\rightarrow f(a) \simeq a$ into $\rightarrow g(f(a)) \simeq h(a), g(a) \simeq h(f(a))$, giving

$$
\text { 10) } \rightarrow g\left(a^{x}\right) \simeq h(a), g(a) \simeq h(f(a))
$$

Here a mark is necessary at the inference position, since $g(f(a)) \nsucc g(a)$ (in fact, we have $g(a) \succ g(f(a)))$. 
A new inference by paramodulation right with 9 into 10 , that is with $\rightarrow \underline{f(a)} \simeq a$ into $\rightarrow g\left(a^{x}\right) \simeq h(a), g(a) \simeq h(f(a))$, gives us

$$
\text { 11) } \rightarrow g\left(a^{x}\right) \simeq h(a), g(a) \simeq h(a)
$$

In this case a mark is not necessary at the inference position, since $h(f(a)) \succ h(a)$.

Now an inference by equality factoring on 11 gives us

$$
\text { 12) } h(a) \simeq h(a) \rightarrow g\left(a^{x}\right) \simeq h(a)
$$

Notice that $g(a) \succ h(a)$, and hence no marking is necessary.

With an inference by equality resolution on 12 , we get

$$
\text { 13) } \rightarrow g\left(a^{x}\right) \simeq h(a)
$$

An inference by paramodulation left with 13 into 3 , that is with $\rightarrow g\left(a^{x}\right) \simeq h(a)$ into $g(x) \simeq h(x) \rightarrow$, gives us

$$
\text { 14) } h(a) \simeq h(a) \rightarrow
$$

Notice that $g(a) \succ h(a)$, and hence no marking is necessary.

Finally, with an inference by equality resolution on 14 the empty clause is obtained.

We must recall that all the markings that we have introduced have only an effect for redundancy purposes. Practical notions of redundancy are addressed in Subsection 5.5.

\subsection{Model Generation}

We use the following multiset extensions to lift the orderings on marked terms to orderings on marked clauses. Let $C$ be a marked clause, and let $\operatorname{emul}(s \cdot \gamma \simeq t \cdot \delta)$ be $\{s \cdot \gamma, t \cdot \delta\}$ if $s \cdot \gamma \simeq t \cdot \delta$ is a positive equation in $C$, and $\{s \cdot \gamma, s \cdot \gamma, t \cdot \delta, t \cdot \delta\}$ if it is negative. Then, if $>$ is an ordering on marked terms, we define the ordering $>^{c}$ on marked clauses by $C>^{c} D$ if $m s e(C)\left(>^{m u l}\right)^{m u l} m s e(D)$, where $m s e(C)$ is the multiset of all emul $(e)$ for occurrences $e$ of equations in $C$. (We analogously lift every equivalence relation $\sim$ on marked terms to an equivalence relation $\sim^{c}$ on clauses.)

Now we define a ground term rewrite system $R_{S}$ from $\operatorname{Forget}(\operatorname{gnd}(S))$, i.e., from the mark-free ground instances of a set of clauses $S$, by induction on $\succ^{c}$, where $\succ$ is the west ordering used in the inference system $\mathscr{G}$. This TRS coincides with the one generated in Bofill et al (2003) for general clauses, where no marks are used.

Definition $13\left(R_{S}\right)$ A clause $C$ of the form $\rightarrow l \simeq r, \Delta$ in Forget $(\operatorname{gnd}(S))$ generates the rule $l \rightarrow r$ in $R_{S}$ if

1. $\left(R_{S}^{C}\right)^{*} \not=C$,

2. $l \succ r$ and $l \simeq r \succ^{m u l} e$ for all equations $e$ in $\Delta$,

3. $l$ is irreducible by $R_{S}^{C}$,

4. $r$ and $\Delta$ are irreducible by $R_{S}^{C}$ at non-topmost positions, and

5. $\left(R_{S}^{C}\right)^{*}=r \simeq r^{\prime}$ for no equation $l \simeq r^{\prime}$ in $\Delta$,

where $R_{S}^{C}$ denotes the set of rules in $R_{S}$ generated by clauses $D$ such that $C \succ^{c} D$. We denote by $R_{S}$ the set of rules generated by all clauses in Forget $(\operatorname{gnd}(S))$. 

$R_{S}$.

As in the equational case, we have the following property which implies convergence of

Property 2 (Bofill et al (2003)) Let $S$ be a set of (marked) clauses. Then for all rules $l \rightarrow r$ in $R_{S}$ we have that

1. $l$ is irreducible by $R_{S} \backslash\{l \rightarrow r\}$, and

2. $r$ is irreducible by $R_{S}$ at non-topmost positions.

Lemma 8 (Bofill et al (2003)) For every set of (marked) clauses $S, R_{S}$ is convergent.

Lemma 9 (Bofill et al (2003)) If a clause $\rightarrow l \simeq r, \Delta$ in Forget $($ gnd $(S))$ generates the rule $l \rightarrow r$ in $R_{S}$, then $\Delta$ is irreducible by $R_{S}$ at non-topmost positions and $R_{S}^{*} \not \models \Delta$.

\subsection{Refutation Completeness}

Here we prove refutation completeness of the inference system $\mathscr{G}$. In order to avoid some problems with lifting arguments, we first restrict to instances with irreducible substitutions, similarly to what is done in Nieuwenhuis and Rubio (1995). However, we only restrict to irreducibility at non-topmost positions.

Definition 14 (Non-Topmost Variable Irreducibility) Let $R$ be a TRS. An instance $C \sigma$ of a marked clause $C$ is said to be non-topmost variable irreducible w.r.t. $R$ if, for all terms $t$ occurring as a side of an equation in Forget $(C)$ and all positions $p$ of $t \sigma$ which are reducible by $R$, we have either $p=\lambda$ or $p$ is a non-variable position of $t$.

Definition 15 (ntirred $\left._{R}(S)\right)$ Let $S$ be a set of marked clauses and $R$ be a TRS. By ntirred $_{R}(S)$ denote the set of all non-topmost variable irreducible ground instances of clauses in $S$ w.r.t. $R$.

Lemma 10 Let $S$ be a set of marked clauses, and $C \sigma$ be a ground instance of a clause $C$ in $S$. If Forget $(C \sigma)$ generates a rule in $R_{S}$, then $C \sigma \in$ ntirred $_{R_{S}}(S)$.

Proof Assume Forget $(C \sigma)$ is of the form $\rightarrow l \simeq r, \Delta$ and the generated rule is $l \rightarrow r$. By Property 2, we have that $l$ and $r$ are irreducible by $R_{S}$ at non-topmost positions and, by Lemma 9, we have that $\Delta$ is irreducible by $R_{S}$ at non-topmost positions. Therefore, for all terms $t$ occurring as a side of an equation in Forget $(C \sigma), t$ is irreducible by $R_{S}$ at nontopmost positions and, hence, $C \sigma \in$ ntirred $_{R_{S}}(S)$.

In the following, we extend the definition of $\succ_{R}$ with an additional case.

Definition $16\left(\succ_{R}\right)$ Let $\succ_{r}$ be a reduction ordering. Let $\succ$ be a west ordering extending $\succ_{r}$. Let $R$ be a terminating ground TRS included in $\succ$ and such that all its right-hand sides are irreducible by $R$ at non-topmost positions. And let $s \cdot \gamma$ and $t \cdot \delta$ be two ground marked terms. Then $s \cdot \gamma \succ_{R} t \cdot \delta$ iff

(i) $s \succ \cup>t$ or

(ii) $s \doteq t$ and $s \gamma \stackrel{+}{\rightarrow}_{R} t \delta$ or

(iii) $s \doteq t, t \delta$ is irreducible by $R$ at non-topmost positions and $s \gamma \succ t \delta$.

In what follows, we will consider $\succ_{R}$ as the transitive closure of the relation defined above (composed with the equivalence relation $\equiv$ on marked terms). 
Property 3 Let $\succ$ be an ordering, and $R$ be a terminating ground TRS included in $\succ$ and such that all its right-hand sides are irreducible by $R$ at non-topmost positions. Then for all ground terms $s$ and $t$ such that $s$ is irreducible by $R$ at non-topmost positions and $s \rightarrow_{R}^{+} t$, we have

1. $s \succ t$ and

2. $t$ is irreducible by $R$ at non-topmost positions.

Proof If $s \rightarrow_{R} s_{1} \rightarrow_{R} \cdots \rightarrow_{R} s_{n} \rightarrow_{R} t$ then, since $s$ is irreducible at non-topmost positions and all right-hand sides of rules in $R$ are irreducible at non-topmost positions, all steps in the sequence are at topmost position, which implies on the one hand that $t$ is irreducible at nontopmost positions and, on the other hand, since $R$ is included in $\succ$, that $s \succ s_{1} \succ \cdots \succ s_{n} \succ t$.

Lemma $11 \succ_{R}$ is well-founded.

Proof By the same arguments as in the proof of Lemma 2 we have that, in an infinite decreasing sequence w.r.t. $\succ_{R}$, there can only be finitely many steps by case (i). Hence, from some point on, there can only be steps by case (ii) or (iii). Now observe that, by Property 3 , after a step by case (iii) all steps by case (ii) are also steps by case (iii). Therefore, if there is an infinite decreasing sequence w.r.t. $\succ_{R}$, there is either an infinite decreasing sequence consisting only of steps by case (ii) or an infinite decreasing sequence consisting only of steps by case (iii). But this contradicts either termination of $R$ or well-foundedness of $\succ$.

The notions of redundancy and saturation are adapted from the ones given in Section 4.2 as follows.

Definition $17\left(\operatorname{ntirred}_{R}(\pi)\right)$ Let $\pi$ be an inference with premises $C_{1}, \ldots, C_{n}$ and conclusion $D$ and $R$ be a TRS. By ntirred $d_{R}(\pi)$ we denote the set all ground instances $\pi \sigma$ of $\pi$ s.t. $C_{1} \sigma, \ldots, C_{n} \sigma$ are non-topmost variable irreducible w.r.t. $R$,

Definition 18 (Redundancy of Inferences) Let $S$ be a set of marked clauses and $R$ a terminating ground TRS. A ground inference by $\mathscr{G}$ with premises $C_{1}, \ldots, C_{n}$ and conclusion $D$ is redundant in $S$ w.r.t. $R$ if we have

$$
\begin{aligned}
& \left.R^{*} \cup \text { Forget } \text { ntirred }_{R}(S)^{\prec_{R}^{c} C_{n}}\right) \\
& \quad \cup \operatorname{Forget}\left(\text { ntirred }_{R}(S) \prec_{R}^{c} D\right) \models \operatorname{Forget}(D) .
\end{aligned}
$$

An inference $\pi$ by $\mathscr{G}$ is redundant in $S$ if for every terminating ground TRS $R$ we have that all inferences in $\operatorname{ntirred}_{R}(\pi)$ are redundant in $S$ w.r.t. $R$.

Definition 19 (Saturatedness) A set $S$ of marked clauses is saturated with respect to $\mathscr{G}$ if every inference by $\mathscr{G}$ with premises in $S$ is redundant in $S$.

Now we are ready to prove refutation completeness of the inference system $\mathscr{G}$. We begin by showing that, if a set of marked clauses $S$ is saturated with respect to $\mathscr{G}$ and $S$ does not contain the empty clause, then $R_{S}^{*}$ is a model of all non-topmost variable irreducible ground instances of $S$ w.r.t. $R_{S}$.

Lemma 12 Let $S$ be a set of marked clauses. If $S$ is saturated with respect to $\mathscr{G}$ and $\square \notin S$, then $R_{S}^{*}=$ Forget $\left(\right.$ ntirred $\left._{R_{S}}(S)\right)$. 
Proof We proceed by induction on $\succ_{R}^{c}$, taking $R=R_{S}$ (recall that $R_{S}$ is a terminating ground TRS that is included in $\succ$ and, by Property 2 , all its right-hand sides are irreducible by $R_{S}$ at non-topmost positions). A contradiction is derived from the existence of a minimal w.r.t. $\succ_{R}^{c}$ ground instance $C \theta$ in ntirred $_{R_{S}}(S)$ of a clause $C$ in $S$ such that $R_{S}^{*} \not \models$ Forget $(C \theta)$.

1. We first consider the case where $C$ is a positive clause $\rightarrow s \cdot \gamma \simeq t \cdot \gamma, \Delta$, where $s \gamma \theta \simeq t \gamma \theta$ is strictly maximal with respect to $\succ^{m u l}$ in $\operatorname{Forget}(C \theta)$ and w.l.o.g. $s \gamma \theta \succ t \gamma \theta$. Since $R_{S}^{*} \not=$ Forget $(C \theta)$, we know that Forget $(C \theta)$ has not generated any rule due to one of the following reasons:

(a) $s \gamma \theta$ is reducible by $R_{S}^{\text {Forget }(C \theta)}$, i.e., condition 3 of Definition 13 fails. Then there exists an instance $C^{\prime} \theta$ of a clause $C^{\prime}$ of the form $\rightarrow l \cdot \gamma \simeq r \cdot \gamma, \Delta_{0}$ in $S$, such that Forget $\left(C^{\prime} \theta\right)$ has generated a rule $l \gamma \theta \rightarrow r \gamma \theta$ reducing $s \gamma \theta$ at some position $p$. There are the following possibilities:

i. Lifting: $\left.s \gamma\right|_{p^{\prime}}$ is a variable $x$ for some prefix $p^{\prime}$ of $p$. In this case, since $C \theta \in$ ntirred $_{R_{S}}(S)$, we necessarily have that $p^{\prime}=p=\lambda$, i.e., $s \gamma=x$ and, moreover, $x$ only occurs at topmost positions in Forget $(C)$.

This in particular means that $s \cdot \gamma$ is either of the form $x^{\prime} \cdot\left\{x^{\prime} \mapsto x\right\}$ or simply $x$, depending on whether $\lambda$ is a marked position of $s \cdot \gamma$ or not. Now let $\theta^{\prime}$ be a ground substitution with the same domain as $\theta$ but where $x \theta^{\prime} \equiv r \gamma \theta$ and $y \theta^{\prime} \equiv$ $y \theta$ for all other variables $y$. This gives us $(s \cdot \gamma) \theta \succ_{R}(s \cdot \gamma) \theta^{\prime}$ by case (i) if $\lambda$ is a non-marked position of $s \cdot \gamma$ (recall that all rules of $R_{S}$ are included in $\succ$ ), and ( $s$. $\gamma) \theta \succ_{R}(s \cdot \gamma) \theta^{\prime}$ by case (ii) (and also by case (iii)) otherwise. Moreover, since $x$ only occurs at topmost positions in Forget $(C)$, we have the same situation in all other equations of $C$ having $x$ in one of their sides. Therefore, we have $C \theta \succ_{R}^{c} C \theta^{\prime}$.

On the other hand, we have that $C \theta^{\prime} \in$ ntirred $_{R_{S}}(S)$, since $C \theta \in$ ntirred $_{R_{S}}(S)$ and, by Property $2, r \gamma \theta$ is irreducible by $R_{S}$ at non-topmost positions.

Finally, since $R_{S}^{*}=l \gamma \theta \simeq r \gamma \theta$ and $R_{S}^{*} \not=\operatorname{Forget}(C \theta)$, then necessarily $R_{S}^{*} \not \models$ Forget $\left(C \theta^{\prime}\right)$, and hence $C \theta^{\prime}$ contradicts the minimality of $C \theta$.

ii. An inference: $p$ is a non-variable position of $s \gamma$. Then there exists an inference $\pi$ by paramodulation right

$$
\frac{\rightarrow l \cdot \gamma \simeq r \cdot \gamma, \Delta_{0} \quad \rightarrow s \cdot \gamma \simeq t \cdot \gamma, \Delta}{\left(\rightarrow s^{\prime} \cdot \gamma^{\prime} \simeq t \cdot \gamma, \Delta_{0}^{\prime}, \Delta\right) \sigma}
$$

where $\sigma=m g u\left(l \gamma,\left.s \gamma\right|_{p}\right)$, according to Definition 12 .

Therefore, the conclusion $D$ of this inference has an instance $D \theta$ of the form $(\rightarrow$ $\left.s^{\prime} \cdot \gamma^{\prime} \simeq t \cdot \gamma, \Delta_{0}^{\prime}, \Delta\right) \theta$ and, moreover, we have that $s^{\prime} \gamma^{\prime} \equiv s \gamma[r \gamma]_{p}$ and $\operatorname{Forget}\left(\Delta_{0}^{\prime}\right)$ is $\operatorname{Forget}\left(\Delta_{0}\right)$.

Now, by the same arguments as in the proof of Lemma 7, we have that $(s$. $\gamma) \theta \succ_{R}\left(s^{\prime} \cdot \gamma^{\prime}\right) \theta$. On the other hand we can prove that, for every equation $u^{\prime}$. $\gamma^{\prime} \simeq v^{\prime} \cdot \gamma^{\prime}$ in $\Delta_{0}^{\prime}$, we have $(s \cdot \gamma \simeq t \cdot \gamma) \theta \succ_{R}^{m u l}\left(u^{\prime} \cdot \gamma^{\prime} \simeq v^{\prime} \cdot \gamma^{\prime}\right) \theta$ :

From Definition 12 we have that, for every equation $u^{\prime} \cdot \gamma^{\prime} \simeq v^{\prime} \cdot \gamma^{\prime}$ in $\Delta_{0}^{\prime}$ there is an equation $u \cdot \gamma \simeq v \cdot \gamma$ in $\Delta_{0}$, where

A. $u^{\prime} \cdot \gamma^{\prime}=u \gamma$ if $s \sigma \succ u \gamma \sigma$ or $t \sigma \succ u \gamma \sigma$,

B. $u^{\prime} \cdot \gamma^{\prime}=u \cdot \gamma$ if the previous case does not apply and $(s \cdot \gamma) \sigma>_{m}(u \cdot \gamma) \sigma$ or $(t \cdot \gamma) \sigma>_{m}(u \cdot \gamma) \sigma$, and

C. $u^{\prime} \cdot \gamma^{\prime}=x \cdot\{x \mapsto u \gamma\}$ for some fresh variable $x$ otherwise, 
and analogously for $v^{\prime} \cdot \gamma^{\prime}$. We consider two possible situations.

Firstly assume that one side of $u^{\prime} \cdot \gamma^{\prime} \simeq v^{\prime} \cdot \gamma^{\prime}$, say $u^{\prime} \cdot \gamma^{\prime}$, is not marked at topmost position. If $u^{\prime} \cdot \gamma^{\prime}=u \gamma$ then there is a side of $s \cdot \gamma \simeq t \cdot \gamma$, say $s \cdot \gamma$, which is not marked at topmost position and $s \sigma \succ u \gamma \sigma$. Then, by stability under substitutions of $\succ$, we have $s \theta \succ u \gamma \theta$, which implies $(s \cdot \gamma) \theta \succ_{R}\left(u^{\prime} \cdot \gamma^{\prime}\right) \theta$ by case (i) of definition of $\succ_{R}$. Analogously, if $u^{\prime} \cdot \gamma^{\prime}=u \cdot \gamma$, there also is a side of $s \cdot \gamma \simeq t \cdot \gamma$, say $s \cdot \gamma$, which is not marked at topmost position and either $(s \cdot \gamma) \sigma>_{m}(u \cdot \gamma) \sigma$ and hence, by stability under substitutions of $>_{m}$, we have $(s \cdot \gamma) \theta>_{m}(u \cdot \gamma) \theta$, which also implies $(s \cdot \gamma) \theta \succ_{R}\left(u^{\prime} \cdot \gamma^{\prime}\right) \theta$ by case (i) of definition of $\succ_{R}$.

In this situation, if $v^{\prime} \cdot \gamma^{\prime}$ is not marked at topmost position then either $(s \cdot \gamma) \theta \succ_{R}$ $\left(v^{\prime} \cdot \gamma^{\prime}\right) \theta$ or $(t \cdot \gamma) \theta \succ_{R}\left(v^{\prime} \cdot \gamma^{\prime}\right) \theta$ for analogous reasons to that of $u^{\prime} \cdot \gamma^{\prime}$ and, if $v^{\prime} \cdot \gamma^{\prime}$ is marked at topmost position, then $(s \cdot \gamma) \theta \succ_{R}\left(v^{\prime} \cdot \gamma^{\prime}\right) \theta$ by case (i) of definition of $\succ_{R}$, since $s \cdot \gamma$ (and hence $\left.(s \cdot \gamma) \theta\right)$ is not marked at topmost position. Altogether, this gives us $(s \cdot \gamma \simeq t \cdot \gamma) \theta \succ_{R}^{m u l}\left(u^{\prime} \cdot \gamma^{\prime} \simeq v^{\prime} \cdot \gamma^{\prime}\right) \theta$.

Secondly assume that both sides of $u^{\prime} \cdot \gamma^{\prime} \simeq v^{\prime} \cdot \gamma^{\prime}$ are marked at topmost position. In this case, if some of $s \cdot \gamma$ or $t \cdot \gamma$ are not marked at topmost position, then we have that $(s \cdot \gamma \simeq t \cdot \gamma) \theta \succ_{R}^{m u l}\left(u^{\prime} \cdot \gamma^{\prime} \simeq v^{\prime} \cdot \gamma^{\prime}\right) \theta$ by case (i) of definition of $\succ_{R}$ as before. If, otherwise, both $s \cdot \gamma$ and $t \cdot \gamma$ are marked at topmost position, then we have that $(s \cdot \gamma \simeq t \cdot \gamma) \theta \succ_{R}^{m u l}\left(u^{\prime} \cdot \gamma^{\prime} \simeq v^{\prime} \cdot \gamma^{\prime}\right) \theta$ by case (iii) of definition of $\succ_{R}$ for the following reasons: (i) $u \gamma \theta$ and $v \gamma \theta$ are irreducible by $R_{S}$ at non-topmost positions by Lemma 9, and (ii) $s \gamma \theta \simeq t \gamma \theta \succ^{m u l} u \gamma \theta \simeq v \gamma \theta$ since $s \gamma \theta \simeq t \gamma \theta$ is strictly maximal w.r.t. $\succ^{m u l}$ in Forget $(C \theta)$ by assumption, $l \gamma \theta \simeq r \gamma \theta$ is strictly maximal w.r.t. $\succ^{m u l}$ in Forget $\left(C^{\prime} \theta\right)$ by construction of $R_{S}$ (and by inference requirements), and Forget $(\boldsymbol{C} \theta) \succ^{c}$ Forget $\left(C^{\prime} \theta\right)$.

Therefore, we have proved that $C \theta \succ_{R}^{c} D \theta$. Moreover, we have that $D \theta \in$ ntirred $_{R_{S}}(S)$, since $C \theta \in$ ntirred $_{R_{S}}(S)$ by assumption and $C^{\prime} \theta \in \operatorname{ntirred}_{R_{S}}(S)$ by Lemma 10 .

Finally, a contradiction is derived from saturatedness of $S$ : On the one hand, since $C \theta \succ_{R}^{c} D \theta$ and $R_{S}^{*} \models$ Forget $\left(\right.$ ntirred $\left._{R_{S}}(S){ }_{R}^{c} C \theta\right)$ by minimality of $C \theta$, we

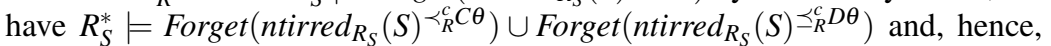
$R_{S}^{*}=\operatorname{Forget}(D \theta)$. On the other hand, since $R_{S}^{*} \not \models \operatorname{Forget}(C \theta)$ we have that $R_{S}^{*} \not \models$ $s \gamma \theta \simeq t \gamma \theta$ and $R_{S}^{*} \not \models$ Forget $(\Delta \theta)$. Then, since $R_{S}^{*}=l \gamma \theta \simeq r \gamma \theta$ and $\left.s \gamma \theta\right|_{p} \equiv l \gamma \theta$, we necessarily have that $R_{S}^{*} \not \models s \gamma[r \gamma]_{p} \theta \simeq t \gamma \theta$, i.e., $R_{S}^{*} \not \models s^{\prime} \gamma^{\prime} \theta \simeq t \gamma \theta$. And moreover $R_{S}^{*} \not \models \operatorname{Forget}\left(\Delta_{0} \theta\right)$ by Lemma 9. Therefore $R_{S}^{*} \not \models$ Forget $(D \theta)$, which is a contradiction.

(b) $t \gamma \theta$ or Forget $(\Delta \theta)$ are reducible by $R_{S}^{\text {Forget }(C \theta)}$ at some non-topmost position, i.e., condition 4 of Definition 13 fails. The proof is like in case 1(a)ii.

(c) None of the previous cases applies and $\Delta$ is of the form $u \cdot \gamma \simeq v \cdot \gamma, \Delta^{\prime}$, where $s \gamma \theta \equiv$ $u \gamma \theta$ and $\left(R_{S}^{\text {Forget }(C \theta)}\right)^{*}=t \gamma \theta \simeq v \gamma \theta$, i.e., condition 5 of Definition 13 fails. Then there is an inference by equality factoring

$$
\frac{\rightarrow s \cdot \gamma \simeq t \cdot \gamma, u \cdot \gamma \simeq v \cdot \gamma, \Delta^{\prime}}{\left(t^{\prime} \cdot \gamma^{\prime} \simeq v \cdot \gamma \rightarrow s \cdot \gamma \simeq t \cdot \gamma, \Delta^{\prime}\right) \sigma}
$$

where $\sigma=m g u(s \gamma, u \gamma), t^{\prime} \cdot \gamma^{\prime}=t \cdot \gamma$ if $u \sigma \succ t \sigma$ or $(u \cdot \gamma) \sigma \gg_{m}(t \cdot \gamma) \sigma$, and $t^{\prime} \cdot \gamma^{\prime}=$ $x \cdot\{x \mapsto t \gamma\}$ for some fresh variable $x$ otherwise.

The conclusion $D$ of this inference has an instance $D \theta$ of the form $\left(t^{\prime} \cdot \gamma^{\prime} \simeq v \cdot \gamma \rightarrow\right.$ $\left.s \cdot \gamma \simeq t \cdot \gamma, \Delta^{\prime}\right) \theta$. Moreover, we have that $(u \cdot \gamma) \theta \succ_{R}\left(t^{\prime} \cdot \gamma^{\prime}\right) \theta$ : 
If $t^{\prime} \cdot \gamma^{\prime}=t \gamma$ and $u \sigma \succ t \gamma \sigma$ then, by stability under substitutions of $\succ$, we have $u \theta \succ t \gamma \theta$ and hence $(u \cdot \gamma) \theta \succ_{R}\left(t^{\prime} \cdot \gamma^{\prime}\right) \theta$ by case (i) of definition of $\succ_{R}$.

If $t^{\prime} \cdot \gamma^{\prime}=t \cdot \gamma$ and $(u \cdot \gamma) \sigma>_{m}(t \cdot \gamma) \sigma$ then, by stability under substitutions of $\gg_{m}$, we have $(u \cdot \gamma) \theta>_{m}(t \cdot \gamma) \theta$ and hence $(u \cdot \gamma) \theta \succ_{R}(t \cdot \gamma) \theta$, i.e., $(u \cdot \gamma) \theta \succ_{R}\left(t^{\prime} \cdot \gamma^{\prime}\right) \theta$, by case (i) of definition of $\succ_{R}$.

If $t^{\prime} \cdot \gamma^{\prime}=x \cdot\{x \mapsto t \gamma\}$ for some fresh variable $x$ and $u \cdot \gamma$ is not marked at topmost position, then $u \cdot \gamma>_{m} t^{\prime} \cdot \gamma^{\prime}$ and hence $(u \cdot \gamma) \theta \succ_{R}\left(t^{\prime} \cdot \gamma^{\prime}\right) \theta$ by case (i) of definition of $\succ_{R}$ as before.

If $t^{\prime} \cdot \gamma^{\prime}=x \cdot\{x \mapsto t \gamma\}$ for some fresh variable $x$ and $u \cdot \gamma$ is also marked at topmost position, then we have that $(u \cdot \gamma) \theta \succ_{R}\left(t^{\prime} \cdot \gamma^{\prime}\right) \theta$ by case (iii) of definition of $\succ_{R}$ for the following reasons: (i) $u \gamma \theta \equiv s \gamma \theta \succ t \gamma \theta \equiv t^{\prime} \gamma^{\prime} \theta$ by assumption, and (ii) $t \gamma \theta$ is irreducible by $R_{S}$ at non-topmost positions, since none of the previous cases applies, and hence $t \gamma \theta$ is irreducible at non-topmost positions by the rules generated by smaller clauses w.r.t. $\succ^{c}$, and no rule generated by a greater clause w.r.t. $\succ^{c}$ can reduce $t \gamma \theta$ at a non-topmost position, since $\succ$ includes the subterm relation.

Therefore we have that $C \theta \succ_{R}^{c} D \theta$. And $D \theta$ is non-topmost variable irreducible w.r.t. $R_{S}$, as so is $C \theta$. Moreover, we have that $R_{S}^{*} \not \models \operatorname{Forget}(D \theta)$, since $R_{S}^{*} \not \models \operatorname{Forget}(C \theta)$ and $R_{S}^{*}=t \gamma \theta \simeq v \gamma \theta$. Then, from saturatedness of $S$ a contradiction is derived as in the previous cases.

2. If $C$ is a positive clause $\rightarrow s \cdot \gamma \simeq t \cdot \gamma, \Delta$, where $s \gamma \theta \simeq t \gamma \theta$ is maximal but not strictly maximal with respect to $\succ^{m u l}$ in Forget $(C \theta)$, then condition 2 of Definition 13 fails. If $s \gamma \theta$ or $t \gamma \theta$ are reducible at some non-topmost position by some rule in $R_{S}^{\text {Forget }(C \theta)}$, then the reasoning of case 1(a)ii applies. Otherwise, the reasoning of case 1c applies.

3. If $C$ is clause $\Gamma, s \cdot \gamma \simeq t \cdot \gamma \rightarrow \Delta$ where $s \gamma \theta \equiv t \gamma \theta$, then there is an inference by equality resolution

$$
\frac{\Gamma, s \cdot \gamma \simeq t \cdot \gamma}{(\Gamma \rightarrow \Delta) \sigma}
$$

where $\sigma=m g u(s \gamma, t \gamma)$. Then we clearly have that $C \theta \succ_{R}^{c} D \theta$, where $D$ denotes the conclusion of the previous inference. This fact, together with the saturatedness assumption, leads to a contradiction as in the other cases.

4. If $C$ is a clause $\Gamma, s \cdot \gamma \simeq t \cdot \gamma \rightarrow \Delta$ where $s \gamma \theta \not t \gamma \theta$, then assume w.l.o.g. that $s \gamma \theta \succ t \gamma \theta$. Now since $R_{S}^{*} \mid \models \overline{\text { Forget }(C \theta)}$ we have that $R_{S}^{*} \models s \gamma \theta \simeq t \gamma \theta$ and, since by Lemma $8 R_{S}$ is convergent, then there exists a rewrite proof of $s \gamma \theta \simeq t \gamma \theta$ with $R_{S}$, that is, $s \gamma \theta$ and $t \gamma \theta$ must rewrite into the same normal form with $R_{S}$. This implies that either $t \gamma \theta$ is reducible at a non-topmost position or else $s \gamma \theta$ is reducible. (Notice that it cannot be the case that the only possible reduction step on $s \gamma \theta \simeq t \gamma \theta$ is at the topmost position of $t \gamma \theta$. By such a step, a new term $u$ is obtained with $t \gamma \theta \succ u$ and $u$ again irreducible at non-topmost positions by Property 2. Since $s \gamma \theta \succ t \gamma \theta$, such a sequence of topmost steps on $t \gamma \theta$ can never produce $s \gamma \theta$ ).

We consider the case where $s \gamma \theta$ is reducible. (The other one is analogous.) Then, as in case 1a, there exists an instance $C^{\prime} \theta$ of a clause $C^{\prime}$ of the form $\rightarrow l \cdot \gamma \simeq r \cdot \gamma, \Delta_{0}$ in $S$, such that Forget $\left(C^{\prime} \theta\right)$ has generated a rule $l \gamma \theta \rightarrow r \gamma \theta$ reducing $s \gamma \theta$ at some position $p$. Then either the lifting argument applies like in case $1(\mathrm{a}) \mathrm{i}$, or else there is an inference by paramodulation left

$$
\frac{\rightarrow l \cdot \gamma \simeq r \cdot \gamma, \Delta_{0} \quad \Gamma, \underline{s \cdot \gamma \simeq t \cdot \gamma} \rightarrow \Delta}{\left(\Gamma, s^{\prime} \cdot \gamma^{\prime} \simeq t \cdot \gamma \rightarrow \Delta_{0}^{\prime}, \Delta\right) \sigma}
$$

where $\sigma=m g u\left(l \gamma,\left.s \gamma\right|_{p}\right)$, which leads to a contradiction analogously to case 1(a)ii. 
Theorem 2 Let $S$ be a set of marked clauses that is saturated with respect to $\mathscr{G}$. Then $\square \in S$ if, and only if, Forget $(S)$ is unsatisfiable.

Proof The left-to-right implication is trivial. For the right-to-left implication we prove that Forget $(S)$ has a model if $\square \notin S$. By Lemma 12 we have that, if $S$ is saturated with respect to $\mathscr{G}$ and $\square \notin S$, then $R_{S}^{*}=$ Forget $\left(\right.$ ntirred $\left._{R_{S}}(S)\right)$. Now, since $R_{S}$ is terminating, for every ground instance $C \sigma$ of a clause $C$ in $S$ there is a ground instance $C \sigma^{\prime}$ in ntirred $_{R_{S}}(S)$ such that $R_{S}^{*} \cup$ Forget $\left(C \sigma^{\prime}\right) \mid=\operatorname{Forget}(C \sigma)$. Finally, since $R_{S}^{*} \models \operatorname{Forget}\left(C \sigma^{\prime}\right)$ then $R_{S}^{*}=\operatorname{Forget}(C \sigma)$ as well.

\subsection{Derivations and Redundancy of Clauses}

Saturatedness has been defined in Section 4.2 from a static point of view, i.e., in terms of redundancy of inferences on a given set of clauses, regardless of how such a set of clauses can be obtained. Here we address the problem of how to compute such saturated sets.

Usually a saturation procedure is modeled by means of a derivation, a sequence of sets of clauses where each set can be obtained from the previous either by adding a logical consequence or by removing some redundant clause, i.e., a sequence $S_{0}, S_{1}, \ldots$ where for each $S_{i+1}$ we have either

1. $S_{i+1}=S_{i} \cup\{C\}$, for some $C$ such that $\operatorname{Forget}\left(S_{i}\right) \models \operatorname{Forget}(C)$, or

2. $S_{i+1}=S_{i} \backslash\{C\}$, for some $C$ which is redundant in $S_{i}$.

In our case, a clause $C$ is defined to be redundant in a set of clauses $S$ if, for every terminating ground TRS $R$ and every ground substitution $\sigma$ such that $C \sigma$ is non-topmost variable irreducible w.r.t. $R$, we have $R^{*} \cup \operatorname{Forget}\left(\operatorname{ntirred}_{R}(S) \prec_{R}^{c} C \sigma\right) \models \operatorname{Forget}(C \sigma)$.

The redundancy notions for inferences presented in Section 4.2 cover the so-called forward redundancy elimination techniques in which, for example, the conclusion of an inference need not be stored if it follows from smaller clauses. On the other hand, redundancy for clauses is intended to cover the so-called backward redundancy elimination techniques in which, for example, an existing clause can be deleted if it follows from smaller ones, including newer clauses that have been generated later on.

It is well known that, when dealing with a total reduction ordering, simplification by rewriting fits into the notion of derivation, since simplification by rewriting can be modeled by first adding the simplified clause and then removing the original one, which has become redundant (see, e.g., Nieuwenhuis and Rubio (2001)). As we show in the following section, simplification at the skeleton of marked terms is possible in our setting and, hence, we strictly improve the result of Bofill et al (2003) about paramodulation with non-total reduction orderings, where compatibility with simplification was not shown at all.

Clauses belonging, from some $i$ on, to all $S_{k}$ with $k>i$, are called persistent. The set of persistent clauses $S_{\infty}$ is formally defined as $S_{\infty}=\cup_{i} \cap_{k>i} S_{k}$. The usual property that states that all non-persistent clauses occurring in a derivation are redundant w.r.t. the persistent ones also holds here.

Lemma 13 Let $S_{0}, S_{1}, \ldots$ be a derivation and let $C$ be a clause in $\left(\cup_{i} S_{i}\right) \backslash S_{\infty}$. Then $C$ is redundant in $S_{\infty}$. 
Proof We need to show that $C$ is redundant in $S_{\infty}$. For this, we assume the contrary and derive a contradiction. Since $C \in\left(\cup_{i} S_{i}\right) \backslash S_{\infty}$, there must be some $S_{j}$ such that $C$ is redundant in $S_{j}$ and, therefore, $C$ is redundant in $\cup_{i} S_{i}$, i.e., for every terminating ground TRS $R$ and every ground substitution $\sigma$ such that $C \sigma$ is non-topmost variable irreducible w.r.t. $R$,

$$
R^{*} \cup \operatorname{Forget}\left(\text { ntirred }_{R}\left(\cup_{i} S_{i}\right)^{\prec}{ }_{R}^{c} C \sigma\right) \models \operatorname{Forget}(C \sigma) \text {. }
$$

On the other hand, if $C$ is not redundant in $S_{\infty}$ we have that, for some terminating ground TRS $R^{\prime}$ and some ground substitution $\sigma^{\prime}$ such that $C \sigma^{\prime}$ is non-topmost variable irreducible w.r.t. $R^{\prime}$,

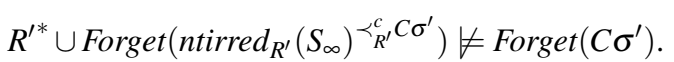

Now let $G$ be the minimal (w.r.t. $\left(\succ_{R^{\prime}}^{c}\right)^{m u l}$ ) finite subset ${ }^{5}$ of ntirred $_{R^{\prime}}\left(\cup_{i} S_{i}\right)^{\prec{ }^{\prime}}{ }^{c} C \sigma^{\prime}$ such that

$$
R^{\prime *} \cup \operatorname{Forget}(G) \models \operatorname{Forget}\left(C \sigma^{\prime}\right) \text {. }
$$

Since $G \nsubseteq$ ntirred $_{R^{\prime}}\left(S_{\infty}\right){ }_{R^{\prime}}^{c} C \sigma^{\prime}$, there is some clause $D$ in $\cup_{i} S_{i}$ which is not persistent and $D \sigma^{\prime \prime} \in G$ for some substitution $\sigma^{\prime \prime}$ (where $D \sigma^{\prime \prime}$ is non-topmost variable irreducible w.r.t. $\left.R^{\prime}\right)$. Then for some $S_{k}$ and some finite subset $G^{\prime}$ of ntirred $_{R^{\prime}}\left(S_{k}\right)^{\prec{ }^{\prime}}{ }^{c} D \sigma^{\prime \prime}$ we have

$$
R^{*} \cup \operatorname{Forget}\left(G^{\prime}\right) \models \operatorname{Forget}\left(D \sigma^{\prime \prime}\right),
$$

and hence

$$
R^{\prime *} \cup \operatorname{Forget}\left(G \backslash\left\{D \sigma^{\prime \prime}\right\} \cup G^{\prime}\right) \models \operatorname{Forget}\left(C \sigma^{\prime}\right),
$$

with $G \backslash\left\{D \sigma^{\prime \prime}\right\} \cup G^{\prime}$ contradicting the minimality of $G$.

In order to obtain a saturated set in the limit of a derivation, some notion of fairness of the derivation is required. We use the following:

Definition 20 (Fairness) A derivation $S_{0}, S_{1}, \ldots$ is fair with respect to an inference system Inf if every inference by Inf using clauses in $S_{\infty}$ is redundant in some $S_{j}$.

This roughly means that all possible inferences have been computed.

Lemma 14 If $S_{0}, S_{1}, \ldots$ is a fair derivation with respect to an inference system Inf, then $S_{\infty}$ is saturated with respect to Inf.

Proof By fairness, every inference $\pi$ using clauses in $S_{\infty}$ is redundant in some $S_{j}$. Then, by Lemma $13, \pi$ is also redundant in $S_{\infty}$. Therefore $S_{\infty}$ is saturated.

Then, from Lemma 14 and Theorem 2, the refutation completeness of any theorem proving procedure which computes a fair derivation w.r.t. the inference system $\mathscr{G}$ follows.

Notice that, in order to ensure fairness in practice, it suffices that no inference with persistent premises is postponed infinitely many times, since adding the conclusion always makes the inference redundant. For example, this could be achieved by periodically considering all inferences with the clause whose size (in number of symbols) is smallest. If a certain clause persists then it will eventually be considered, since there are only finitely many clauses with smaller size.

Finally, let us mention that as a potentially useful strategy, we can restart from time to time the saturation process after removing all the marks we have in our set of clauses. This can be done since all our inference rules are sound with or without marks.

\footnotetext{
5 This finite subset exists by compactness of first order logic.
} 
5.5 Practical Notions of Redundancy

Here we describe in detail practical notions for redundancy of inferences, which include techniques for forward simplification. All these techniques can be straightforwardly adapted to redundancy of clauses, e.g., backward simplification, by using clauses smaller than the clause to be redunded instead of clauses smaller than the maximal premise of the inference.

We describe three forms of redundancy that can be used in practice. They all have been implemented in our prototype (see Section 7). The first one is deletion by subsumption where, differently to the standard subsumption, we have to be careful with the marked positions. The other two methods use simplification by rewriting. In the first case, we remove the marks of the clause to be simplified, allowing for more simplification, but we have to explicitly check whether the rules in use (which only include equations that can be oriented by the reduction ordering) are smaller than the maximal premise of the inference. In the second case, we keep the marks. This reduces the amount of allowed simplification, but it is more efficient since in most cases there is no need to check if the rule in use is smaller than the maximal premise.

Lemma 15 Let $S$ be a set of marked clauses and $D \cdot \gamma$ a marked clause. If there is some clause $D^{\prime} \cdot \delta$ in $S$ such that, for some substitution $\sigma, D \gamma \supseteq D^{\prime} \delta \sigma, D \cdot \gamma \geq_{m}^{c}\left(D^{\prime} \cdot \delta\right) \sigma$ and $x \sigma \in \mathscr{X}$ for all $x \in \operatorname{Var}\left(D^{\prime}\right) \backslash \operatorname{Dom}(\delta)$ then

- any inference $\pi$ with premises in $S$ and conclusion $D \cdot \gamma$ is redundant in $S$.

- $D \cdot \gamma$ is redundant in $S$, provided that $D^{\prime} \cdot \delta \not \equiv D \cdot \gamma$.

Note that, since substitutions are only defined for non-marking variables, the conditions on $\sigma$ mean that for every variable $x$ in $D^{\prime} \delta$ (i.e., forgetting the marks of $D^{\prime} \cdot \delta$ ) either it is instantiated with a variable in $D \gamma$ (i.e., forgetting the marks of $D \cdot \gamma$ as well) or it can only occur below marks in $D^{\prime} \cdot \delta$. This is roughly because redundancy is defined using (nontopmost) variable irreducible instances w.r.t. $R$ which are smaller w.r.t. $\succ_{R}$, but rewriting (i.e., normalizing) with $R$ only is guaranteed to provide smaller instances w.r.t. $R$ if the rewrite steps take place below marks.

Proof We show that $R^{*} \cup$ Forget $\left(\right.$ ntirred $\left._{R}\left(\left\{D^{\prime} \cdot \delta\right\}\right)_{R}^{c}(D \cdot \gamma) \rho\right) \models D \gamma \rho$ for every terminating ground TRS $R$ and for every ground substitution $\rho$ such that $(D \cdot \gamma) \rho$ is non-topmost variable irreducible w.r.t. $R$.

We have $D \cdot \gamma \geq_{m}^{c}\left(D^{\prime} \cdot \delta\right) \sigma$ and hence, by stability under substitutions of $\geq_{m}^{c}$, we also have $(D \cdot \gamma) \rho \geq_{m}^{c}\left(D^{\prime} \cdot \delta\right) \sigma \rho$, i.e., $\left.D \rho \cdot(\gamma \circ \rho)\right|_{D o m}(\gamma) \geq\left._{m}^{c} D^{\prime} \sigma \rho \cdot(\delta \circ \sigma \circ \rho)\right|_{D o m(\delta)}$. Therefore $D \rho \supseteq{ }^{c} D^{\prime} \sigma \rho$ and moreover, since $D \gamma \supseteq D^{\prime} \delta \sigma$ and hence $D \gamma \rho \supseteq D^{\prime} \delta \sigma \rho$, we have $(D \cdot \gamma) \rho \succeq_{R}^{c}\left(D^{\prime} \cdot \delta\right) \sigma \rho$. Now, since $\left(D^{\prime} \cdot \delta\right) \sigma \rho$ may be non-topmost variable reducible w.r.t. $R$, we have to show that otherwise there is a smaller instance that is non-topmost variable irreducible.

Let $\sigma^{\prime}$ be the normalization w.r.t. $R$ of $\rho \cup(\sigma \circ \rho)$ for all variables $x$ in $D^{\prime} \delta$ that occur at some non-topmost position, i.e., $x \sigma^{\prime}=x \sigma \rho$ if $x$ occurs in $D^{\prime} \delta$ only at topmost positions, and $x \sigma^{\prime}$ is a normal form w.r.t. $R$ of $x \sigma \rho$ otherwise. Then $\left(D^{\prime} \cdot \delta\right) \sigma^{\prime}$ is trivially non-topmost variable irreducible w.r.t. $R$, and $R^{*} \cup\left\{D^{\prime} \delta \sigma^{\prime}\right\}=D \gamma \rho$. Now we show that $\left(D^{\prime} \cdot \delta\right) \sigma \rho \succeq_{R}^{c}$ $\left(D^{\prime} \cdot \delta\right) \sigma^{\prime}$. As said, for every variable $x$ in occurring in $D^{\prime} \delta$ either

1. it is instantiated with a variable $y$ in $D \gamma$, and hence $x \sigma \rho \equiv y \rho$, or else

2. it can only occur below marks in $D^{\prime} \cdot \delta$. 
Now, in the first case, if $y$ occurs in $D \gamma$ at some non-topmost position then $y \rho$ is irreducible w.r.t. $R$, and hence $x \sigma^{\prime} \equiv x \sigma \rho \equiv y \rho$. If, otherwise, $y$ occurs in $D \gamma$ only at topmost positions then, since $D \gamma \supseteq D^{\prime} \delta \sigma$, we have that $x$ occurs in $D^{\prime} \delta$ only at topmost positions, and hence $x \sigma^{\prime} \equiv x \sigma \rho \equiv y \rho$.

Concluding, since $x \sigma \rho \rightarrow_{R}^{*} x \sigma^{\prime}$ if $x$ only occurs below marks in $D^{\prime} \cdot \delta$ and $x \sigma^{\prime} \equiv x \sigma \rho$ otherwise, we have $D^{\prime} \sigma \rho \doteq D^{\prime} \sigma^{\prime}$ and $D^{\prime} \delta \sigma \rho \rightarrow_{R}^{*} D^{\prime} \delta \sigma^{\prime}$ and hence $\left(D^{\prime} \cdot \delta\right) \sigma \rho \succeq_{R}^{c}\left(D^{\prime} \cdot \delta\right) \sigma^{\prime}$.

Example 4 Let $\pi$ be an inference with premises in $S$ and conclusion $\rightarrow f(a, y) \simeq g(y)$. Then $\pi$ is redundant in $S$ if, e.g., there is some clause in $S$ of the form:

1. $\rightarrow f(a, x) \simeq g(x)$

2. $(\rightarrow f(z, x) \simeq g(x)) \cdot\{z \mapsto a\}$

3. $\left(\rightarrow f\left(z_{1}, z_{2}\right) \simeq g\left(z_{2}\right)\right) \cdot\left\{z_{1} \mapsto a, z_{2} \mapsto x\right\}$

4. $\left(\rightarrow f\left(z_{1}, z_{2}\right) \simeq z_{3}\right) \cdot\left\{z_{1} \mapsto a, z_{2} \mapsto x, z_{3} \mapsto g(x)\right\}$

5. $\left(\rightarrow f\left(z_{1}, z_{2}\right) \simeq z_{3}\right) \cdot\left\{z_{1} \mapsto x_{1}, z_{2} \mapsto x_{2}, z_{3} \mapsto x_{3}\right\}$

However, $\rightarrow f(z, x) \simeq g(x)$ does not subsume $\rightarrow f(a, y) \simeq g(y)$ since, in our setting, nonmarking variables occurring at the skeleton can only be instantiated with variables.

Regarding the use of subsumption in the context of redundancy of clauses, the only point to be mentioned is that, if we try to find a clause $D^{\prime}$ in $S \backslash D$ that subsumes $D$ using the same conditions as in the lemma above then we directly have that $D>{ }_{m}^{c} D^{\prime}$ (as it cannot be equal), and hence we can show that the clause is redundant using the same proof.

Now we address the techniques for simplification. We introduce the following relation to compare clauses. It is used to guarantee that the rules in use are smaller enough.

Definition 21 ( $\succ_{\text {red }}$ ) Let $\succ$ be a west ordering and $s \cdot \gamma$ and $t \cdot \delta$ be two marked terms. Then $s \cdot \gamma \succ_{\text {red }} t \cdot \delta$ iff $s \succ t$ or $s \cdot \gamma>_{m} t \cdot \delta$.

In what follows we ambiguously denote by $\succ_{\text {red }}$ the transitive closure of this relation composed with the equivalence on marked terms.

Note that, by stability under substitutions of $\succ$ and $>_{m}$ we trivially have that $\succ_{\text {red }}$ is stable under substitutions. Moreover, since $s \cdot \gamma>_{m} t \cdot \delta$ implies $s \gg t$ (see Definition 2), if $s \cdot \gamma \succ_{\text {red }} t \cdot \delta$ we have $s \succ U>t$ and, since $\succ U>$ is well-founded (see proof of Lemma 2) so is $\succ_{\text {red }}$. Moreover, for ground marked terms, $\succ_{\text {red }}$ is included in $\succ_{R}$ for every terminating ground TRS $R$, since it coincides with case (i) of $\succ_{R}$.

As an example of application of this ordering, assuming $f(x) \succ g(x)$ for all $x$, then $h(f(a)) \succ_{\text {red }} f(a) \succ_{\text {red }} f\left(a^{x}\right) \succ_{\text {red }} g\left(a^{x}\right) \succ_{\text {red }} b^{x}$, since $h(f(a)) \triangleright f(a)>f(x) \succ g(x) \triangleright x$ (recall that $\triangleright$ is included in every west ordering $\succ$ ).

Lemma 16 Let $s \cdot \gamma, t \cdot \delta, u \cdot \rho$ and $v \cdot \theta$ be marked terms. If $s \cdot \gamma \doteq_{m} t \cdot \delta \succ_{\text {red }} u \cdot \rho \doteq_{m} v \cdot \theta$ then $s \cdot \gamma \succ_{\text {red }} v \cdot \theta$.

Proof There are two cases depending on $t \cdot \delta \succ_{\text {red }} u \cdot \rho$. If we have $t \cdot \delta>_{m} u \cdot \rho$ then we have $s \cdot \gamma \doteq_{m} t \cdot \delta>_{m} u \cdot \rho \doteq_{m} v \cdot \theta$, which implies $s \cdot \gamma>_{m} v \cdot \theta$. Otherwise we have $t \succ u$. Moreover, since $s \cdot \gamma \doteq_{m} t \cdot \delta$, we have $s \sigma \equiv t$ for some variable renaming substitution $\sigma$ on marking variables. Analogously, since $u \cdot \rho \doteq_{m} v \cdot \theta, u \equiv v \phi$ for some variable renaming substitution $\phi$ on marking variables, and hence $s \sigma \succ v \phi$. Now let $\gamma^{\prime}$ be the substitution such that $x \sigma \gamma^{\prime}=x \gamma$ for all $x \in \operatorname{Dom}(\gamma)$, and $\theta^{\prime}$ be the substitution such that $x \phi \theta^{\prime}=x \theta$ for all $x \in \operatorname{Dom}(\theta)$. Then $s \cdot \gamma \equiv s \sigma \cdot \gamma^{\prime}, s \sigma \cdot \gamma^{\prime} \succ_{\text {red }} v \phi \cdot \theta^{\prime}$ (since $s \sigma \succ v \phi$ ) and $v \phi \cdot \theta^{\prime} \equiv v \cdot \theta$, which implies $s \cdot \gamma \succ_{\text {red }} v \cdot \theta$ by definition. 
As said at the beginning of this section, in the first technique for simplification we forget the marks of the clause to be simplified. In that case, we are forced to check that the equations we are using are smaller than the maximal premise of the inference. This can cause some inefficiency in the redundancy process, but on the other hand once we know that the equation is smaller we can use it almost freely. However in order to make the process feasible in practice we require to apply the equations in a reductive way, that is, at any position if the equation is included in the reduction ordering or at topmost position if it is only included in the west ordering. Additionally, we need a condition on the instantiation of the variables similar to the one imposed for applying subsumption.

Definition 22 Let $E$ be a set of equations and $t$ be a non-marked term. Then $t \Rightarrow_{E} t^{\prime}$ if there is an equation $e_{1} \cdot \delta_{1} \simeq e_{2} \cdot \delta_{2}$ in $E$ and a substitution $\sigma$ such that either

1. $e_{1} \delta_{1} \sigma \succ_{r} e_{2} \delta_{2} \sigma,\left.t\right|_{p} \equiv e_{1} \delta_{1} \sigma$ and $t^{\prime} \equiv t\left[e_{2} \delta_{2} \sigma\right]_{p}$, or

2. $e_{1} \delta_{1} \sigma \succ e_{2} \delta_{2} \sigma, t \equiv e_{1} \delta_{1} \sigma$ and $t^{\prime} \equiv e_{2} \delta_{2} \sigma$,

and for all $x \in \operatorname{Var}\left(e_{1}\right) \backslash \operatorname{Dom}\left(\delta_{1}\right)$ we have $x \sigma \in \mathscr{X}$.

Notice that the requirement on the instantiation implies that non-marking variables occurring at the skeleton can only be instantiated with variables.

We ambiguously write $C \Rightarrow_{E} C^{\prime}$ for clauses, if some term $t$ occurring in $C$ is rewritten as defined.

Lemma 17 Let $S$ be a set of clauses, $E$ be a set of marked equations (i.e., positive unit clauses) in $S$, and $\pi$ be an inference with rightmost premise $C$ and conclusion $D$. If

- $\operatorname{Forget}(D) \Rightarrow_{E}^{+} D^{\prime}$,

$-C \succ_{\text {red }}^{c} e_{1} \cdot \delta_{1} \simeq e_{2} \cdot \delta_{2}$ for every equation in $E$, and

- $D^{\prime}$ is a tautology or there is a clause $D^{\prime \prime}$ in $S$ such that $C \succ_{\text {red }}^{c} D^{\prime \prime}$ and Forget $\left(D^{\prime \prime}\right) \equiv D^{\prime}$,

then $\pi$ is redundant in $S$.

Note that using this lemma if $D^{\prime}$ is a tautology we can infer that $\pi$ is redundant in $S$. Moreover if $D^{\prime}$ is not a tautology and there is no such $D^{\prime \prime}$ in $S$, we can create it and then we have that $\pi$ is redundant in $S \cup\left\{D^{\prime \prime}\right\}$.

Proof We will show that for every terminating ground TRS $R$ and every ground substitution $\rho$ such that $C \rho$ and $D \rho$ are non-topmost variable irreducible w.r.t. $R$, we have that $R^{*} \cup$ Forget $\left._{\text {ntirred }}\left(E \cup\left\{D^{\prime \prime}\right\}\right)^{\prec c} C \rho\right) \models$ Forget $(D \rho)$.

We prove, by induction on the length of the derivation, that for all non-marked clauses $D_{1}$ and ground substitutions $\rho$ such that $D_{1} \rho$ is non-topmost variable irreducible w.r.t. $R$, if $D_{1} \Rightarrow_{E}^{+} D_{n}$ then we have that $D_{n} \rho$ is non-topmost variable irreducible w.r.t. $R$ and $R^{*} \cup$ ntirred $_{R}(E) \prec_{R}^{c} C \rho \cup\left\{D_{n} \rho\right\} \models D_{1} \rho$. Notice that, with this result, taking $D_{1}=\operatorname{Forget}(D)$ and $D_{n}=D^{\prime}=\operatorname{Forget}\left(D^{\prime \prime}\right)$, we can conclude that $R^{*} \cup$ Forget $\left._{\text {ntirred }}\left(E \cup\left\{D^{\prime \prime}\right\}\right)^{\prec}{ }_{R}^{c} C \rho\right) \models$ Forget $(D \rho)$, since $D^{\prime \prime} \rho$ is non-topmost variable irreducible w.r.t. $R$ (as it is $D^{\prime} \rho$ ) and by assumption $C \succ_{\text {red }}^{c} D^{\prime \prime}$ and hence, by stability under substitutions of $\succ_{\text {red }}$ and inclusion of $\succ_{\text {red }}$ in $\succ_{R}$ for ground marked terms, $C \rho \succ_{R}^{c} D^{\prime \prime} \rho$.

We have to prove that if $D_{1} \rho$ is non-topmost variable irreducible w.r.t. $R$ and $D_{1} \Rightarrow_{E} D_{2}$ then $D_{2} \rho$ is non-topmost variable irreducible w.r.t. $R$ and $R^{*} \cup \operatorname{irred}_{R}(E){ }_{R}^{c} C \rho \cup\left\{D_{2} \rho\right\} \models$ $D_{1} \rho$. If we do so, then we are done since if $n>2$, then by induction we have that $D_{n} \rho$ is non-topmost variable irreducible w.r.t. $R$ and $R^{*} \cup \operatorname{irred}_{R}(E){ }_{R}^{c} C \rho \cup\left\{D_{n} \rho\right\} \mid=D_{2} \rho$, which allows us to conclude that $R^{*} \cup \operatorname{irred}_{R}(E) \prec_{R}^{c} C \rho \cup\left\{D_{n} \rho\right\} \models D_{1} \rho$. 
Let us prove the property we claimed. Let $t$ be the (non-marked) term $t$ in $D_{1}$ where the rewriting step $D_{1} \Rightarrow_{E} D_{2}$ is applied with an equation $e_{1} \cdot \delta_{1} \simeq e_{2} \cdot \delta_{2}$ and substitution $\theta$, with $x \theta \in \mathscr{X}$ for all $x \in \operatorname{Var}\left(e_{1}\right) \backslash \operatorname{Dom}\left(\delta_{1}\right)$. There are two cases:

1. $e_{1} \delta_{1} \theta \succ_{r} e_{2} \delta_{2} \theta,\left.t\right|_{p} \equiv e_{1} \delta_{1} \theta$ and $t^{\prime} \equiv t\left[e_{2} \delta_{2} \theta\right]_{p}$, or

2. $e_{1} \delta_{1} \theta \succ e_{2} \delta_{2} \theta, t \equiv e_{1} \delta_{1} \theta$ and $t^{\prime} \equiv e_{2} \delta_{2} \theta$.

Since $\succ_{r}$ and $\succ$ are well-founded and stable under substitutions, we have $\operatorname{Var}\left(e_{1} \delta_{1} \theta\right) \supseteq$ $\operatorname{Var}\left(e_{2} \delta_{2} \theta\right)$. Therefore, if $D_{1} \Rightarrow_{E} D_{2}$ then $\operatorname{Var}\left(D_{1}\right) \supseteq \operatorname{Var}\left(D_{2}\right)$ and we cannot rewrite at any variable position, and thus if a variable is at topmost position in $D_{1}$ then it is at topmost position in $D_{2}$. Therefore if $D_{1} \rho$ is non-topmost variable irreducible w.r.t. $R$ then $D_{2} \rho$ also is.

Now, since $\left.t\right|_{p} \equiv e_{1} \delta_{1} \theta$ and $\left.t^{\prime}\right|_{p} \equiv e_{2} \delta_{2} \theta$ implies $\left.t\right|_{p} \rho \equiv e_{1} \delta_{1} \theta \rho$ and $\left.t^{\prime}\right|_{p} \rho \equiv e_{2} \delta_{2} \theta \rho$, we have that $\left\{\left(e_{1} \delta_{1} \simeq e_{2} \delta_{2}\right) \theta \rho, D_{2} \rho\right\} \mid=D_{1} \rho$. However, $\left(e_{1} \cdot \delta_{1} \simeq e_{2} \cdot \delta_{2}\right) \theta \rho$ might be nontopmost variable reducible w.r.t. $R$. To overcome this problem we build a new substitution $\rho^{\prime}$ such that

1. $\left(e_{1} \cdot \delta_{1} \simeq e_{2} \cdot \delta_{2}\right) \rho^{\prime}$ is non-topmost variable irreducible w.r.t. $R$,

2. $R^{*} \cup\left\{\left(e_{1} \delta_{1} \simeq e_{2} \delta_{2}\right) \rho^{\prime}\right\} \models\left(e_{1} \delta_{1} \simeq e_{2} \delta_{2}\right) \theta \rho$, and

3. either $\rho^{\prime}=\theta \circ \rho$ or $\left(e_{1} \cdot \delta_{1} \simeq e_{2} \cdot \delta_{2}\right) \theta \rho \succ_{R}^{m u l}\left(e_{1} \cdot \delta_{1} \simeq e_{2} \cdot \delta_{2}\right) \rho^{\prime}$.

Let $\rho^{\prime}$ be the normalization w.r.t. $R$ of $\theta \circ \rho$, i.e., $x \rho^{\prime} \equiv x \theta \rho \downarrow_{R}$ for all $x \in \operatorname{Var}\left(e_{1} \delta_{1}\right)$. Then the first two conditions above trivially hold. Let us show the third one.

First, as said, we have that $\operatorname{Var}\left(e_{1} \delta_{1} \theta\right) \supseteq \operatorname{Var}\left(e_{2} \delta_{2} \theta\right)$ and $e_{1} \delta_{1} \theta \notin \mathscr{X}$ due to $e_{1} \delta_{1} \theta \succ$ $e_{2} \delta_{2} \theta$ (recall that $\succ_{r}$ is included in $\succ$ ).

Second, since $\left.t\right|_{p} \equiv e_{1} \delta_{1} \theta$ and $x \theta \in \mathscr{X}$ for all $x \in \operatorname{Var}\left(e_{1}\right) \backslash \operatorname{Dom}\left(\delta_{1}\right)$ (and so for all $x \in \operatorname{Var}\left(e_{2}\right) \backslash \operatorname{Dom}\left(\delta_{2}\right)$ ), we have that $x \boldsymbol{\theta} \in \operatorname{Var}(t)$ for all non-marking variables $x$ of $e_{1} \cdot \delta_{1} \simeq$ $e_{2} \cdot \delta_{2}$ in $\operatorname{Var}\left(e_{1}\right) \cup \operatorname{Var}\left(e_{2}\right)$. Now, since $t$ is non-topmost variable irreducible and $t$ cannot be a variable, for all non-marking variables $x$ of $e_{1} \cdot \delta_{1} \simeq e_{2} \cdot \delta_{2}$ in $\operatorname{Var}\left(e_{1}\right) \cup \operatorname{Var}\left(e_{2}\right)$ we have $x \theta \rho$ is irreducible by $R$, and hence $x \rho^{\prime} \equiv x \theta \rho$.

Therefore, if $x \rho^{\prime} \not \equiv x \theta \rho$ for some variable $x$ then $x$ only occurs below marked positions in $e_{1} \cdot \delta_{1} \simeq e_{2} \cdot \delta_{2}$, which implies that either $\rho^{\prime}=\theta \circ \rho$ or $\left(e_{1} \cdot \delta_{1} \simeq e_{2} \cdot \delta_{2}\right) \theta \rho \succ_{R}^{m u l}\left(e_{1} \cdot \delta_{1} \simeq\right.$ $\left.e_{2} \cdot \delta_{2}\right) \rho^{\prime}$ using case (ii) of $\succ_{R}$.

Finally, by assumption we have $C \succ_{\text {red }}^{c} e_{1} \cdot \delta_{1} \simeq e_{2} \cdot \delta_{2}$, which by stability under substitutions of $\succ_{\text {red }}$ implies $C \rho \succ_{\text {red }}^{c}\left(e_{1} \cdot \delta_{1} \simeq e_{2} \cdot \delta_{2}\right) \theta \rho$ and, from the fact that $\succ_{\text {red }}$ is included in $\succ_{R}$ for ground marked terms, implies $C \rho \succ_{R}^{c}\left(e_{1} \cdot \delta_{1} \simeq e_{2} \cdot \delta_{2}\right) \theta \rho$. Then from this, the fact that $\left\{\left(e_{1} \delta_{1} \simeq e_{2} \delta_{2}\right) \theta \rho, D_{2} \rho\right\} \mid=D_{1} \rho$ and the three conditions above we have that $R^{*} \cup$ irred $_{R}(E)^{\prec{ }_{R}^{c} C \rho} \cup\left\{D_{2} \rho\right\} \mid=D_{1} \rho$.

Finally, we consider simplification by rewriting of the conclusion of an inference but keeping the marks, i.e., by rewriting in a more standard way. We first introduce the notion of marked rule and marked rewriting.

Definition 23 (Marked rule) A marked rule, denoted by $(l \rightarrow r) \cdot \chi$ or simply $l \rightarrow r \cdot \chi$, is an equation $l \cdot \chi \simeq r \cdot \chi$ such that $\operatorname{Dom}(\chi) \subseteq \operatorname{Var}(l), \operatorname{Ran}(\chi) \subseteq \mathscr{X}, \operatorname{Var}(l) \cap \operatorname{Ran}(\chi)=\emptyset$ and $l \succ_{r} r$.

We say that $l \rightarrow r \cdot \chi$ is in a set of clauses $S$ if the equation $l \cdot \chi \simeq r \cdot \chi$ belongs to $S$.

We are using the Greek letter $\chi$ to denote the substitution of the marked left and righthand side of the marked rule to graphically recall that the only subterms that can be marked are the variables in $\mathscr{X}$. Note also that the condition $\operatorname{Var}(l) \cap \operatorname{Ran}(\chi)=\emptyset$ implies that no 
variable in $\mathscr{X}$ can occur both marked and non-marked in the rule. Finally note that from $l \succ_{r} r$, since $\succ_{r}$ is a reduction ordering, we have $\operatorname{Var}(r) \subseteq \operatorname{Var}(l)$ and hence $\operatorname{Var}(r \chi) \subseteq$ $\operatorname{Var}(l \chi)$.

Definition 24 (Marked rewriting) Let $l \rightarrow r \cdot \chi$ be a marked rule and $t \cdot \gamma$ be a marked term. Let $p$ be a non-variable position of $t$ and $\sigma$ be a substitution s.t. $\left.l \chi \sigma \equiv t\right|_{p}$ and for all $x \in \operatorname{Var}(l) \backslash \operatorname{Dom}(\chi)$ we have $x \sigma \in \operatorname{Var}(t) \backslash \operatorname{Dom}(\gamma)$. Then $t \cdot \gamma \rightarrow_{l \rightarrow r \cdot \chi}^{p} t[r \chi \sigma]_{p} \cdot \gamma$.

If $M R$ is a set of marked rules, then $\rightarrow_{M R}$ denotes one step rewriting with a rule in $M R$.

Note that if $t$ has no marks and $t \rightarrow_{M R} t^{\prime}$ then $t^{\prime}$ has no marks either. Moreover, the last condition of the definition implies that non-marking variables in the left-hand side of the rule can only be instantiated with variables. This has to do with the use of variable irreducible instances in all completeness proofs.

Let us show you several examples.

Example 5 Consider the following rules.

1. $f(x, x) \rightarrow h(x) \cdot\left\{x \mapsto x^{\prime}\right\}$.

2. $f(g(x, y), a) \rightarrow f(a, y) \cdot\left\{x \mapsto x^{\prime}, y \mapsto y^{\prime}\right\}$.

3. $f(g(x, y), a) \rightarrow f(a, y) \cdot\left\{x \mapsto x^{\prime}\right\}$.

4. $f(g(x, y), a) \rightarrow f(a, y)$.

Using the first rule we have $h(f(a, a)) \rightarrow h(h(a))$ and $h(f(g(z), g(z))) \cdot\{z \mapsto a\} \rightarrow$ $h(h(g(z))) \cdot\{z \mapsto a\}$, but $h\left(f\left(z_{1}, z_{2}\right)\right) \cdot\left\{z_{1} \mapsto a, z_{2} \mapsto a\right\}$ cannot be rewritten since there is no matching of $f(x, x)$ on $f\left(z_{1}, z_{2}\right)$.

Using the second rule we have $f(g(h(z), a), a) \cdot\{z \mapsto a\} \rightarrow f(a, a)$, but we cannot rewrite $f(z, a) \cdot\{z \mapsto g(h(a), a)\}$.

Using the third rule we can rewrite the term $h\left(f\left(g\left(h(z), z^{\prime}\right), a\right)\right) \cdot\{z \mapsto a\}$ into $h\left(f\left(a, z^{\prime}\right)\right)$. However, we cannot rewrite this term with the last rule, since it would imply instantiating a non-marking variable with a non-variable term.

Lemma 18 Let $l \rightarrow r \cdot \chi$ be a marked rule. If $s \cdot \gamma \rightarrow_{l \rightarrow r \cdot \chi}^{p} t \cdot \gamma$ then $s \succ t$.

Proof From the definition of marked rewriting we have that $l \chi \sigma=\left.s\right|_{p}$ and $t=s[r \chi \sigma]_{p}$ for some substitution $\sigma$. Moreover, from the definition of marked rule we have that $l \succ_{r} r$. Then, since $\succ_{r}$ is included in $\succ$ and is monotonic and stable under substitutions, we have that $s=s[l \chi \sigma]_{p} \succ s[r \chi \sigma]_{p}=t$, i.e., $s \succ t$.

As said, the following lemmas show that although we can rewrite in less positions, in most cases we do not need to check that the rules we are using are smaller than the maximal premise.

Lemma 19 Let $l \rightarrow r \cdot \chi$ be a marked rule. If $s \cdot \gamma \rightarrow_{l \rightarrow r \cdot \chi}^{p} t \cdot \gamma$ with substitution $\sigma$ then $\left.s \gamma\right|_{p} \equiv l \chi \sigma \gamma$ and either $(i) s \cdot \gamma \succ_{\text {red }}(l \cdot \chi) \sigma \gamma$ or $(i i) s \cdot \gamma \equiv(l \cdot \chi) \sigma \gamma$.

Proof By definition of marked rewriting we have that $\left.s\right|_{p} \equiv l \chi \sigma$. Hence $\left.\left.s\right|_{p} \gamma \equiv s \gamma\right|_{p} \equiv l \chi \sigma \gamma$. Now we show that either (i) $s \cdot \gamma \succ_{\text {red }}(l \cdot \chi) \sigma \gamma$ or (ii) $s \cdot \gamma \equiv(l \cdot \chi) \sigma \gamma$. Since $\left.s\right|_{p} \equiv l \chi \sigma$ and for all $x \in \operatorname{Var}(l) \backslash \operatorname{Dom}(\chi)$ we have $x \sigma \in \operatorname{Var}\left(\left.s\right|_{p}\right) \backslash \operatorname{Dom}(\gamma)$, then $\left.s\right|_{p} \cdot \gamma \geq_{m}(l \cdot \chi) \sigma \dot{\doteq}_{m}(l \cdot \chi) \sigma \gamma$. Now, if $p \neq \lambda$ then $\left.s \cdot \gamma \succ_{\text {red }} s\right|_{p} \cdot \gamma$. In this case, if $\left.s\right|_{p} \cdot \gamma>_{m}(l \cdot \chi) \sigma \gamma$ then $\left.s\right|_{p} \cdot \gamma \succ_{\text {red }}(l \cdot \chi) \sigma \gamma$ and hence $s \cdot \gamma \succ_{\text {red }}(l \cdot \chi) \sigma \gamma$. Otherwise, if $\left.s\right|_{p} \cdot \gamma \doteq_{m}(l \cdot \chi) \sigma \gamma$ then $s \cdot \gamma \succ_{\text {red }}(l \cdot \chi) \sigma \gamma$ by Lemma 16. For the case where $p=\lambda$, we have $s \cdot \gamma \geq_{m}(l \cdot \chi) \sigma \gamma$. If $s \cdot \gamma>_{m}(l \cdot \chi) \sigma \gamma$ then $s \cdot \gamma \succ_{\text {red }}(l \cdot \chi) \sigma \gamma$. If $s \cdot \gamma \dot{=}_{m}(l \cdot \chi) \sigma \gamma$ then, since $s \gamma \equiv l \chi \sigma \gamma$, we have $s \cdot \gamma \equiv(l \cdot \chi) \sigma \gamma$. 
In what follows we will precisely describe how we can apply forward simplification using marked rewriting.

Lemma 20 Let $S$ be a set of marked clauses, MR be a set of marked rules in $S$ and $\pi$ be an inference with maximal premise $C$ and conclusion $D$. If

- $D \rightarrow{ }_{M R}^{+} D^{\prime}$ and

- for every topmost rewriting step on an original term $s \cdot \gamma$ in $D$ that occurs only in positive literals with a rule $l \rightarrow r \cdot \chi$ and substitution $\theta$ such that $s \cdot \gamma \equiv(l \cdot \chi) \theta \gamma$, we have that $C \succ_{\text {red }}^{c}(l \cdot \chi \simeq r \cdot \chi) \theta \gamma$

then $\pi$ is redundant in $S \cup\left\{D^{\prime}\right\}$.

Notice that if $D^{\prime}$ is a tautology we have that $\pi$ is redundant in $S$. By an original term in $D$ we mean a term that has not been introduced by a previous rewriting step.

Proof Let $\sigma$ be the mgu of the inference. We will show that for every terminating ground TRS $R$ and every ground substitution $\rho=\sigma \circ \sigma^{\prime}$ such that $C \rho$ and $D \rho$ are non-topmost variable irreducible w.r.t. $R$, we have that (i) $D \rho \rightarrow_{M R}^{+} D^{\prime} \rho$ using rules in $M R$ with ground instances following from $R^{*} \cup$ ntirred $_{R}(M R)^{\prec}{ }_{R}^{c} C \rho$, (ii) $C \rho \succ_{\text {red }}^{c} D^{\prime} \rho$ (which implies $C \rho \succ_{R}^{c}$ $D^{\prime} \rho$ ) and (iii) $D^{\prime} \rho$ is non-topmost variable irreducible w.r.t. $R$. Therefore, we conclude $R^{*} \cup$ Forget $\left._{\text {ntirred }}(M R)_{R} \prec_{R}^{c} C \rho\right) \cup \operatorname{Forget}\left(\right.$ ntirred $\left._{R}\left(\left\{D^{\prime}\right\}\right) \prec_{R}^{c} C \rho\right) \models \operatorname{Forget}(D \rho)$, and thus $\pi$ is redundant in $S \cup\left\{D^{\prime}\right\}$.

We proceed by induction on the length of the derivation. Assume we have $D=D_{1} \rightarrow_{M R}^{*}$ $\cdots D_{k} \rightarrow_{M R} D^{\prime}$. Then, by induction hypothesis, if $k>1$ we have (i), (ii) and (iii) for $D_{k}$. Therefore, we have to show that $D_{k} \rightarrow_{M R} D^{\prime}$ preserves all three properties. Assume the step is applied on a term $s \cdot \gamma$ in $D_{k}$ with a rule $l \rightarrow r \cdot \chi$. Then $\left.l \chi \theta \equiv s\right|_{p}$ for some position $p$ and substitution $\theta$, and $s \cdot \gamma \rightarrow_{l \rightarrow r \cdot \chi} s[r \chi \theta]_{p} \cdot \gamma$. Taking $\theta^{\prime}=\left.(\theta \circ \rho)\right|_{\operatorname{Var}(l)}$ we have that $l \theta^{\prime} \rightarrow r \theta^{\prime} \cdot \chi$ is a marked rule with the same marking variables as $l \rightarrow r \cdot \chi$ (note that, since $\theta$ is always applied after $\chi$ we assume that $\operatorname{Dom}(\theta) \cap \operatorname{Dom}(\chi)=\emptyset)$. Moreover, since $\theta^{\prime}$ is restricted to (a subset of) the variables of $l$ and $\operatorname{Var}(l) \cap \operatorname{Ran}(\chi)=\emptyset$, we have that $l \theta^{\prime} \rightarrow r \theta^{\prime} \cdot \chi$ corresponds to the instance $(l \cdot \chi \simeq r \cdot \chi) \theta^{\prime}$ of the equation $l \cdot \chi \simeq r \cdot \chi$ in $S$. And, since $\left.l \chi \theta \rho \equiv s \rho\right|_{p}$, then $\left.l \chi \theta^{\prime \prime} \equiv s \rho\right|_{p}$ for some $\theta^{\prime \prime}$ extending $\theta^{\prime}$ for the variables in $\operatorname{Ran}(\chi)$, and hence $\left.\left.(s \cdot \gamma) \rho \equiv s \rho \cdot(\gamma \circ \rho)\right|_{\operatorname{Dom}(\gamma)} \rightarrow_{l \theta^{\prime} \rightarrow r \theta^{\prime} \cdot \chi} s \rho\left[r \chi \theta^{\prime \prime}\right]_{p} \cdot(\gamma \circ \rho)\right|_{\operatorname{Dom}(\gamma)}$.

By Lemma 18 we have that $s \rho \succ s \rho\left[r \chi \theta^{\prime \prime}\right]_{p}$, and thus $\left.s \rho \cdot(\gamma \circ \rho)\right|_{D o m(\gamma)} \succ_{\text {red }} s \rho\left[r \chi \theta^{\prime \prime}\right]_{p}$. $\left.(\gamma \circ \rho)\right|_{\operatorname{Dom}(\gamma)}$, which implies $D_{k} \rho \succ_{\text {red }}^{c} D^{\prime} \rho$. If $k=1$ then $D_{k}=D$. Since $C \rho$ and $D \rho$ are nontopmost variable irreducible instances w.r.t. $R$ then, by definition of the inference rules and the proof of Lemma 12, we have that either $C \rho \doteq_{m} D \rho$ or $C \rho \succ_{\text {red }}^{c} D \rho$ (using the fact that $\succ_{\text {red }}$ coincides with case (i) of $\succ_{R}$ for ground marked terms). Then, by Lemma 16, it holds (ii) $C \rho \succ_{\text {red }}^{c} D^{\prime} \rho$. Otherwise, if $k>1$, by induction hypothesis we have $C \rho \succ_{\text {red }}^{c} D_{k} \rho$, and hence (ii) holds as well.

Now we show that (iii) holds, i.e., $D^{\prime} \rho$ is non-topmost variable irreducible w.r.t. $R$. Since by definition of marked rule it follows that $\operatorname{Var}(l \chi) \supseteq \operatorname{Var}(r \chi)$, we have $\operatorname{Var}\left(s[l \chi \theta]_{p}\right) \supseteq$ $\operatorname{Var}\left(s[r \chi \theta]_{p}\right)$ and hence $\operatorname{Var}\left(\operatorname{Forget}\left(D_{k}\right)\right) \supseteq \operatorname{Var}\left(\operatorname{Forget}\left(D^{\prime}\right)\right)$. Moreover, we have that $p$ is a non-variable position of $s$, and thus if a variable is at topmost position in $D_{k}$ then it is at topmost position in $D^{\prime}$. Therefore if $D_{k} \rho$ is non-topmost variable irreducible w.r.t. $R$ then $D^{\prime} \rho$ also is. If $k=1$ then by assumption $D_{k} \rho$ is non-topmost variable irreducible w.r.t. $R$. Otherwise, we have it by induction hypothesis.

Finally, to prove that (i) is preserved, we will show that $(l \cdot \chi \simeq r \cdot \chi) \phi \rho$ follows from $R^{*} \cup$ ntirred $_{R}(M R)^{\prec c} C \rho$, where $\phi=\theta^{\prime \prime} \circ \gamma$. Notice that $l \chi \theta^{\prime \prime} \gamma \rho \simeq r \chi \theta^{\prime \prime} \gamma \rho$ is ground since $\left.l \chi \theta^{\prime \prime} \gamma \rho \equiv s \rho \gamma \rho\right|_{p}$ and $\operatorname{Var}(l) \supseteq \operatorname{Var}(r)$. Moreover, since $\theta^{\prime \prime}$ is an extension of $\theta^{\prime}$ for the variables in $\operatorname{Ran}(\chi)$, we also have that $(l \cdot \chi \simeq r \cdot \chi) \phi \rho \equiv^{m u l}\left(l \theta^{\prime} \cdot \chi \simeq r \theta^{\prime} \cdot \chi\right) \phi \rho$. And, as 
seen before, we have $D_{k} \rho \rightarrow_{l \theta^{\prime} \rightarrow r \theta^{\prime} \cdot \chi} D^{\prime} \rho$. Hence, if we prove that $(l \cdot \chi \simeq r \cdot \chi) \phi \rho$ follows from $R^{*} \cup$ ntirred $_{R}(M R){ }_{R}^{c} C \rho$ we can conclude that we have (i) for $D_{k}$.

We first show that $C \rho \succ_{\text {red }}^{c}(l \cdot \chi \simeq r \cdot \chi) \phi \rho$, which implies $C \rho \succ_{R}^{c}(l \cdot \chi \simeq r \cdot \chi) \phi \rho$ since $\succ_{\text {red }} \subseteq \succ_{R}$ for ground instances. If $s \cdot \gamma$ is an original term in $D$ that occurs only in positive literals, $p=\lambda$ and $s \cdot \gamma \equiv(l \cdot \chi) \theta \gamma$, then we have $C \succ_{\text {red }}^{c}(l \cdot \chi \simeq r \cdot \chi) \theta \gamma$ by assumption, which implies $C \rho \succ_{\text {red }}^{c}(l \cdot \chi \simeq r \cdot \chi) \theta \gamma \rho$ by stability under substitutions of $\succ_{\text {red }}$. Now observe that $(l \cdot \chi \simeq r \cdot \chi) \theta \gamma \rho \equiv(l \cdot \chi \simeq r \cdot \chi) \theta \rho \gamma \rho$ since $\operatorname{Dom}(\rho) \cap \operatorname{Dom}(\gamma)=\emptyset$ and $\rho$ is ground. Moreover $(l \cdot \chi \simeq r \cdot \chi) \theta \rho \gamma \rho \equiv{ }^{m u l}(l \cdot \chi \simeq r \cdot \chi) \theta^{\prime \prime} \gamma \rho$ since $l \theta \rho \equiv l \theta^{\prime \prime}$ and $\operatorname{Var}(l) \supseteq \operatorname{Var}(r)$. Finally, since $(l \cdot \chi \simeq r \cdot \chi) \theta^{\prime \prime} \gamma \rho \equiv^{m u l}(l \cdot \chi \simeq r \cdot \chi) \phi \rho$, we get $C \rho \succ_{\text {red }}^{c}(l \cdot \chi \simeq r \cdot \chi) \phi \rho$ as desired.

Otherwise we prove that $D \rho \succ_{r e d}^{c}(l \cdot \chi \simeq r \cdot \chi) \phi \rho$ and since, as seen before, either $C \rho \dot{\triangle}_{m}$ $D \rho$ or $C \rho \succ_{\text {red }}^{c} D \rho$, we have $C \rho \succ_{\text {red }}^{c}(l \cdot \chi \simeq r \cdot \chi) \phi \rho$. There are three cases depending on the rewriting step and the term that is rewritten:

- $s \cdot \gamma$ is not original, i.e., there is some term $u \cdot \delta$ in $D$ s.t. $u \cdot \delta \rightarrow_{M R}^{+} s \cdot \gamma$. Then by Lemma 18 we have $u \succ s$ and hence $u \cdot \delta \succ_{\text {red }} s \cdot \gamma$. Moreover, by Lemma 19 either $s \cdot \gamma \succ_{\text {red }}(l$. $\chi) \theta \gamma$ or $s \cdot \gamma \equiv(l \cdot \chi) \theta \gamma$ and, as seen before, $(l \cdot \chi \simeq r \cdot \chi) \theta \rho \gamma \rho \equiv^{m u l}(l \cdot \chi \simeq r \cdot \chi) \phi \rho$. Therefore, by stability under substitutions, $(u \cdot \delta) \rho \succ_{\text {red }}(l \cdot \chi) \phi \rho$. And, since $l \succ_{r} r$, we also have $(u \cdot \delta) \rho \succ_{\text {red }}(r \cdot \chi) \phi \rho$, which implies $D \rho \succ_{\text {red }}^{c}(l \cdot \chi \simeq r \cdot \chi) \phi \rho$.

- $s \cdot \gamma$ occurs in a negative literal in $D$. Then, as in the previous case, we have that either $(s \cdot \gamma) \rho \succ_{\text {red }}(l \cdot \chi) \phi \rho$ or $(s \cdot \gamma) \rho \equiv(l \cdot \chi) \phi \rho$, and $(s \cdot \gamma) \rho \succ_{\text {red }}(r \cdot \chi) \phi \rho$, which implies $D \rho \succ_{\text {red }}^{c}(l \cdot \chi \simeq r \cdot \chi) \phi \rho$ by definition of $\succ_{\text {red }}^{c}$ on negative literals.

- $s \cdot \gamma$ is original in $D$ and either $p \neq \lambda$, or $p=\lambda$ but $s \cdot \gamma \not \equiv(l \cdot \chi) \theta \gamma$. Then $s \cdot \gamma \succ_{\text {red }}(l \cdot \chi) \theta \gamma$ either from the fact that the strict subterm relation is included in $\succ$ or by Lemma 19 . Therefore $(s \cdot \gamma) \rho \succ_{\text {red }}(l \cdot \chi) \phi \rho$, and moreover $(l \cdot \chi) \phi \rho \succ_{\text {red }}(r \cdot \chi) \phi \rho$ since $l \succ_{r} r$, which lets us conclude that $D \rho \succ_{\text {red }}^{c}(l \cdot \chi \simeq r \cdot \chi) \phi \rho$.

Finally, since $(l \cdot \chi \simeq r \cdot \chi) \phi \rho$ might be non-topmost variable reducible w.r.t. $R$, as in the proof of Lemma 17 we build a substitution $\rho^{\prime}$ in a way such that

1. $(l \cdot \chi \simeq r \cdot \chi) \rho^{\prime}$ is non-topmost variable irreducible w.r.t. $R$,

2. $R^{*} \cup\left\{(l \cdot \chi \simeq r \cdot \chi) \rho^{\prime}\right\} \mid=(l \cdot \chi \simeq r \cdot \chi) \phi \rho$, and

3. either $\rho^{\prime}=\phi \circ \rho$ or $(l \cdot \chi \simeq r \cdot \chi) \phi \rho \succ_{R}^{m u l}(l \cdot \chi \simeq r \cdot \chi) \rho^{\prime}$.

The construction and the proof are analogous. Then, we have that $(l \cdot \chi \simeq r \cdot \chi) \rho^{\prime} \in$ ntirred $_{R}(M R) \prec_{R}^{c} C \rho$ and thus $(l \cdot \chi \simeq r \cdot \chi) \phi \rho$ follows from $R^{*} \cup$ ntirred $_{R}(M R){ }^{\prec_{R}^{c} C \rho}$, which allows us to conclude.

Note that before using a rule for marked rewriting we have to mark (some of) its variable positions. This can be done adding the marked version and removing the original because it has become redundant.

Lemma 21 Let $S$ be a set of clauses and let $s \cdot \chi \simeq t \cdot \chi$ be a marked rule such that $s^{\prime} \simeq$ $t^{\prime} \dot{ }^{m u l} s \simeq t$ and $s^{\prime} \simeq t^{\prime}>_{m}{ }^{m u l} s \cdot \chi \simeq t \cdot \chi$ for some equation $s^{\prime} \simeq t^{\prime}$ in $S$. Then $s^{\prime} \simeq t^{\prime}$ is redundant in $S \backslash\left\{s^{\prime} \simeq t^{\prime}\right\} \cup\{s \cdot \chi \simeq t \cdot \chi\}$.

Proof It holds easily since, on the one hand, from $s^{\prime} \simeq t^{\prime} \dot{=}^{m u l} s \simeq t$ and $\operatorname{Ran}(\chi) \subseteq \mathscr{X}$ we have $s^{\prime} \simeq t^{\prime} \dot{\doteq}^{m u l} s \chi \simeq t \chi$ and, on the other hand, from $s^{\prime} \simeq t^{\prime}>_{m}{ }^{m u l} s \cdot \chi \simeq t \cdot \chi$ we have $\left(s^{\prime} \simeq t^{\prime}\right) \rho \succ_{R}(s \cdot \chi \simeq t \cdot \chi) \rho$ for all ground instances.

Let us make some remarks. 
- As we have seen, due to the fact that we can only use irreducible instances, we need to mark some variables before applying a rewriting step. Moreover, since we need the skeleton left-hand side of the rule to be larger than the skeleton right hand side, we mark all occurrences of the same variable using the same marking variable.

- We are only using equations without marks for rewriting, which are then marked, if necessary, in some positions where variables occur. The reason to only consider equations without marks is because every time we add a mark in the conclusion of an inference, we introduce a new fresh variable that replaces a subterm, and thus it is hardly possible that this kind of equations can be oriented with the reduction ordering comparing only the skeleton of the left and the right-hand side.

Marks forbidding simplification are introduced in order to, roughly, compatibilize paramodulation steps using non-reductive equations and simplification steps using reductive ones. In the following example we show that demodulation w.r.t. $\succ_{r}$ can cause incompleteness if no marks are introduced at all, when redundancy with smaller equations w.r.t. the subterm relation is also considered.

Example 6 Let $\succ_{r}$ be a reduction including

$$
\begin{aligned}
& f(x, x) \succ_{r} f(a, b) \text { and } \\
& f(x, x) \succ_{r} f(b, a) .
\end{aligned}
$$

Notice that then necessarily

$$
\begin{aligned}
& a \nsucc_{r} b \text { and } \\
& b \nsucc_{r} a .
\end{aligned}
$$

Let $S$ denote the following inconsistent set of Horn clauses:

$$
\begin{aligned}
& \text { 1) } \rightarrow a \simeq b \\
& \text { 2) } \rightarrow f(x, x) \simeq f(a, b) \\
& \text { 3) } \rightarrow f(x, x) \simeq f(b, a) \\
& \text { 4) } \rightarrow f(x, x) \simeq f(b, b) \\
& \text { 5) } \rightarrow g(f(a, b)) \simeq g(g(f(b, a))) \\
& \text { 6) } x \simeq g(x) \rightarrow
\end{aligned}
$$

First of all, we will show how the empty clause can be derived under our inference system (which introduces the convenient marks). Let $\succ$ be a west ordering which is an extension of $\succ_{r}$ and that includes

$$
\begin{aligned}
g(g(f(a, b))) & \succ g(f(a, b)) \succ g(g(f(b, a))) \succ g(f(b, a)) \\
\succ f(a, a) & \succ f(b, b) \succ f(a, b) \succ f(b, a) \succ a \succ b .
\end{aligned}
$$

An inference by paramodulation right with 1 into 5 is possible, giving

$$
\text { 7) } \rightarrow g\left(f\left(b^{x_{0}}, b\right)\right) \simeq g(g(f(b, a)))
$$

A mark at the inference position is necessary since $g(f(a, b)) \nsucc g(f(b, b))$ (recall that $\left.f(x, x) \succ_{r} f(a, b)\right)$. 
A new inference by paramodulation right with 1 into 7 gives us

$$
\text { 8) } \rightarrow g\left(f\left(b^{x_{0}}, b\right)\right) \simeq g\left(g\left(f\left(b, b^{x_{1}}\right)\right)\right)
$$

In this case a mark is necessary at the inference position since $g(g(f(b, a))) \nsucc g(g(f(b, b)))$ (recall that $f(x, x) \succ_{r} f(b, a)$ ).

Now, since $\succ$ includes the subterm relation, we have that $g(g(f(b, b))) \succ g(f(b, b))$ and hence an inference by paramodulation left with 8 into 6 is possible, giving

$$
\text { 9) } g\left(f\left(b, b^{x_{1}}\right)\right) \simeq g\left(f\left(b^{x_{0}}, b\right)\right) \rightarrow
$$

Finally, the empty clause is obtained with an inference by equality resolution.

Now let us show that, if the marks were not introduced, then refutation completeness could be lost in a fair saturation procedure:

Inferences by paramodulation right with 1 into 5 would give either

$$
\rightarrow g(f(b, b)) \simeq g(g(f(b, a)))
$$

or

$$
\rightarrow g(f(a, b)) \simeq g(g(f(b, b))),
$$

if no marks were introduced. Then, these clauses could be simplified back into 5 , respectively, with 2 and 3 (which are included in the reduction ordering). Moreover, inferences by paramodulation right with 1 into 2 and 3 would give us 4 . And inferences by paramodulation right between 2, 3 and 4 would either give us already existing clauses, or clauses like

$$
\rightarrow f(a, b) \simeq f(b, a),
$$

which follow from the smaller (w.r.t. the subterm relation) clause 1. Finally, since we have $g(f(a, b)) \succ g(g(f(b, a)))$, an inference by paramodulation left with 5 into 6 would give us

$$
\text { 7) } f(a, b) \simeq g(g(f(b, a))) \rightarrow
$$

but, since $f(a, b) \simeq g(g(f(b, a)))$ does not follow from the positive equations, further inferences on 7 would not lead to the empty clause. Therefore the set $S$ could be saturated without the empty clause being derived.

This example therefore shows that the marks that we introduce are not merely a technicality to prove completeness.

\section{Knuth-Bendix Completion}

Let $E$ denote a set of equations and $\succ_{r}$ denote a (possibly non-totalizable) reduction ordering on $T(\mathscr{F}, \mathscr{X})$. Then a convergent TRS $R$ for $E$ and $\succ_{r}$ is a convergent TRS, logically equivalent to $E$, and such that $l \succ_{r} r$ for all its rules $l \rightarrow r$.

Finding a convergent TRS for the given $E$ and $\succ_{r}$ whenever it exists, and finding it in finite time if it is finite, is a well-known problem. Although this can be done by enumerating all equational consequences of $E$, finding practically useful procedures remained as an open problem for a long time. In Bachmair et al (1989) an unfailing completion procedure is given, for the case that the ordering $\succ_{r}$ is total on $E$-equivalent terms. Also, Devie showed that for left- and right linear $E$ (i.e., no variable occurs more than once in a side of an equation) standard Knuth-Bendix completion finds $R$ (Devie (1990)). But it was not 
until in Bofill et al $(1999,2003)$ that a procedure for the general case, not relying on the enumeration of all equational consequences, was presented.

From our current result, it directly follows that the method of Bofill et al (2003) can be made compatible (to some amount) with simplification by rewriting. In this method we need to consider an interreduced ground TRS for the model, since we prove that a canonical (i.e., interreduced) convergent TRS $R$ for a set of equations $E$ is included in the persistent set of equations obtained from $E$. That is, we are not interested in any convergent TRS $R$ for $E$, but in a canonical convergent TRS $R$ for $E$, which is minimal and unique. As in Bofill et al (2003), we just need to use the inference system of Definition 4, but where the paramodulation inference is relaxed by changing condition 3 as follows:

3. for some ground substitution $\theta$, we have $l \delta \sigma \theta \succ r \delta \theta$.

With this change, now the ordered paramodulation rule is also applied on top of the small sides, and hence we obtain an interreduced ground TRS for the model. We denote the resulting inference system by $\mathscr{K}$.

Moreover, since an equation $s \simeq t$ can be identified with a clause $\rightarrow s \simeq t$, the redundancy notions for clauses of Section 5.4 hold for equations as well and, hence, simplification by rewriting is possible by using marked rewrite rules. Then, by the same arguments as in Bofill et al (2003) we get the following result.

Theorem 3 Let $E_{0}, E_{1}, \ldots$ be a fair derivation with respect to $\mathscr{K}$, where $E_{0}$ is the given set of equations $E$. Then Forget $\left(E_{\infty}\right) \supseteq R$, where $R$ is a (canonical) convergent TRS for $E$ and $\succ_{r}$.

Therefore, if $R$ is finite, it will be contained in $\operatorname{Forget}\left(E_{j}\right)$ for some $E_{j}$ of the derivation.

\section{Experiments}

In order to check if our ideas are feasible at least for small examples we have developed a prototype written in Prolog that implements for the equational case the inference system given in Section 4 and the practical notions of redundancy described in Section 5.5. We have completed automatically all equational examples given in the paper up to this point, plus some variants (including some more equations). The implementation and the examples are available at http://www.lsi.upc.edu/ albert/marking.zip. In this section we illustrate how our prototype works, and give an additional example that cannot be saturated by our system.

In our prototype, the ordering is defined by means of pairs of terms that are said to be ordered by the reduction ordering or by the west ordering. It is the user's duty to check whether the given ordering is altogether a west ordering. In the future we plan to allow the user the definition of the ordering by means of a semantic path ordering (as described in Section 8).

As an example, we show how an equational version of Example 6 can be saturated by our system. The input file should be as shown in Figure 1, by following Prolog syntax for predicates and terms (recall that all sentences must be finished with '.' and that variables start with capital letter). Sentences starting with '\%' are treated as comments.

There are two predicates that can be used to define the west-ordering:

gr (+Term, +Term).

ge (+Term, +Term). 
The first one defines a strict decreasing comparison between two terms. The second one defines a non-strict comparison.

There are also two predicates that can be used to define a reduction ordering that will be assumed to be included in the west-ordering (i.e., they extend the definitions given by the predicates above):

$\operatorname{grr}(+$ Term, + Term $)$.

ger $(+$ Term, + Term).

As before, the first one defines a strict decreasing comparison between two terms, and the second one defines a non-strict comparison.

The reduction ordering will be finally obtained by using the monotonic extension of all instances of the given definitions. The final west-ordering is obtained as the union of the reduction ordering, the subterm relation and all instances of the given definitions.

After the ordering, the initial set of equations is given by repeatedly using the predicate:

eq $(+$ Term, + Term $)$.

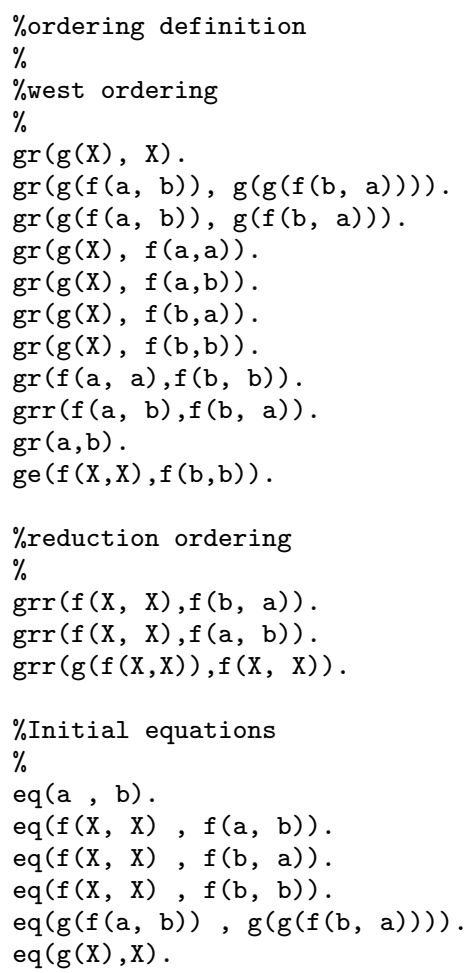

Fig. 1 Example of input file.

When saturating a set of equations, first of all, the system checks whether some of the equations can be oriented with respect to the reduction ordering, which allows us to make 
some simplification initially. For instance, for the case of the example of Figure 1, we get the messages shown in Figure 2.

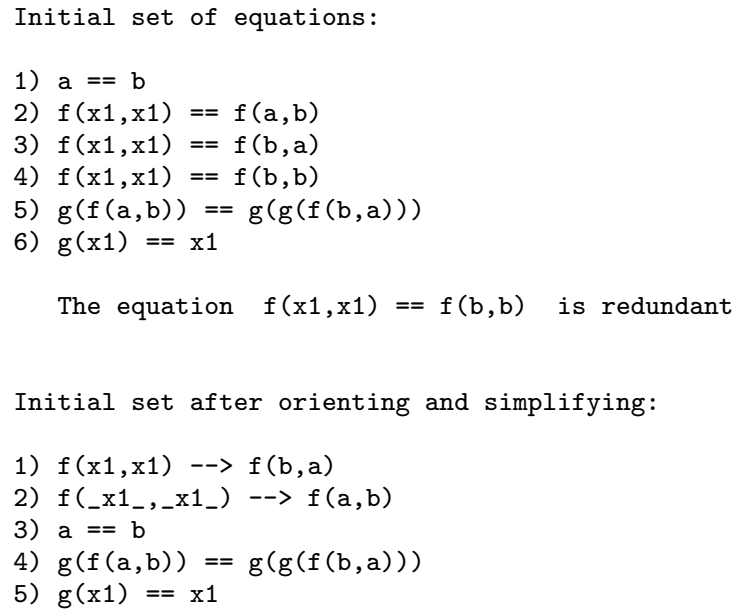

Fig. 2 Initial set of equations after orienting and simplifying.

Notice that both sides of the equation $f(x, x)=f(b, b)$ can be rewritten with the rule $f(x, x) \rightarrow f(a, b)$ (which is included in the reduction ordering and smaller) into $f(a, b)$, leading to a tautology, and hence $f(x, x)=f(b, b)$ can be removed. Notice also that, according to the requirements for marked rewriting (see Definition 24), the variables of the left-hand side of the rewrite rule $f(x, x) \rightarrow f(a, b)$ must be marked in order to be able to rewrite the term $f(b, b)$, as non-marking variables can only be instantiated with variables. The marks are represented with underscores in the output as in $f\left({ }_{-} \mathrm{x} 1_{-},{ }_{-} \mathrm{x} 1_{-}\right)-->\mathrm{f}(\mathrm{b}, \mathrm{a})$. In fact, after marking the variables, the non-marked version of the same equation becomes redundant in the presence of the marked one.

After obtaining the initial set of equations, the system starts the saturation process, by performing paramodulation inferences according to the inference system given in Section 4 and applying all the practical redundancy notions described in Section 5.5. During this process, the system outputs messages like the ones in Figure 3. If the saturation process terminates, then it outputs the final set of equations (see Figure 4).

Often, differently marked versions of the same equation appear. For instance, in Figure 4 we have both $f(a, b)-->f(b, a)$ and $f_{-}(a, b)_{-}==_{-} f(b, a)_{-}$, that is, the same equation but with marks at top level in both sides (which becomes unorientable). Our experiments show that if the initial set contains equations that can be oriented with the reduction ordering then we can apply a reasonable amount of simplification. For this reason, it is important to avoid subsuming equations that are oriented by the reduction ordering using equations that are not (as in the previous example). In fact, sometimes this is crucial to complete the set of equations.

We remark that, both for technical reasons and as shown in a counterexample (Example 6) the addition of some marks in the terms under certain circumstances ${ }^{6}$ is needed in

\footnotetext{
6 Stated in detail in the definitions of our inference systems and our practical notions of redundancy.
} 


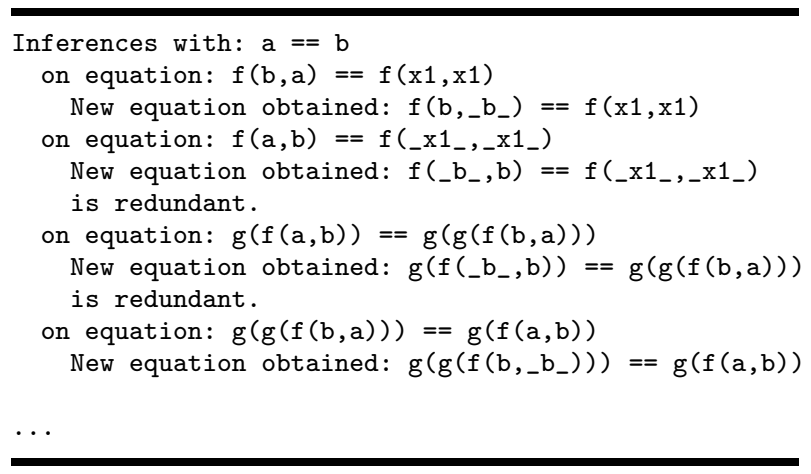

Fig. 3 Messages during the saturation process.

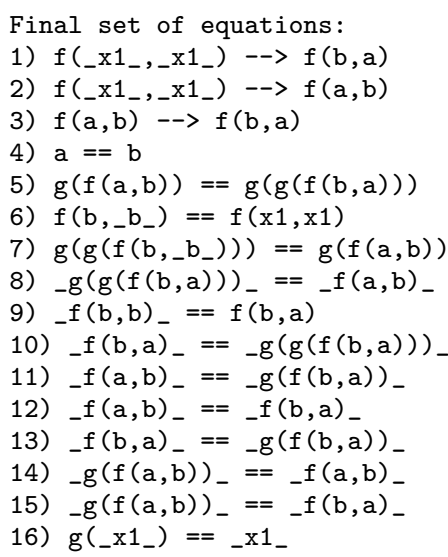

Fig. 4 Final set of equations.

order to preserve refutation completeness. As explained throughout the paper, these marks have the effect of diminishing the amount of redundancy, as marked subterms are treated as variables for redundancy purposes. The drawback is that, in some cases, those marks may prevent the procedure from saturating the set of clauses.

For instance, the completion of Example 1 described in the introduction is possible under our system, as Figure 5 shows. Notice that the variable $x$ of the rewrite rule $h(x) \rightarrow$ $f(g(x))$ used to simplify must be marked, as it is instantiated with a non-variable term when rewriting. But the system is already closed since no inference is possible.

However, the following syntactically similar instance cannot be successfully completed under our inference system.

Example 7 Let us consider the single equation

$$
f f(x) \simeq f g f(x)
$$

where $f f(x) \succ_{r} f g f(x)$ for some reduction ordering $\succ_{r}$. Notice that this ordering cannot be totalized without losing monotonicity, since $f(x)$ and $g f(x)$ should be incomparable in any 
The west ordering $>$ is defined as the transitive closure of:

$f(f(g(x 1)))>f(g(f(g(x 1))))$

$h(x 1)>r f(g(x 1))$

$h(f(g(x 1)))>r f(f(g(x 1)))$

where $>r$ defines a reduction ordering included in >

Initial set of equations:

1) $h(x 1)==f(g(x 1))$

2) $h(f(g(x 1)))==f(f(g(x 1)))$

The equation $h(f(g(x 1)))==f(f(g(x 1)))$

has been simplified into $f(g(f(g(x 1))))==f(f(g(x 1)))$

Initial set after orienting and simplifying:

1) $\mathrm{h}\left(\right.$ ( $\left.\mathrm{x} 1_{-}\right) \rightarrow \mathrm{f}\left(\mathrm{g}\left(\mathrm{x} \mathbf{1}_{-}\right)\right)$

2) $f(g(f(g(x 1))))==f(f(g(x 1)))$

Inferences with: $h\left({ }_{-} \mathrm{x} 1_{-}\right)==\mathrm{f}\left(\mathrm{g}\left({ }_{-} \mathrm{x} 1_{-}\right)\right)$

Inferences with: $f(g(f(g(x 1))))==f(f(g(x 1)))$

Final set of equations:

1) $h\left(x 1_{1}\right) \rightarrow f\left(g\left(x 1_{-}\right)\right)$

2) $f(g(f(g(x 1))))==f(f(g(x 1)))$

Fig. 5 Completion of Example 1 under our system.

monotonic extension, for analogous reasons as in Example 1. First of all we show that, for every possible inference with this equation, the conclusion can be simplified into a tautology. By an ordered paramodulation with

$$
f f(x) \simeq f g f(x)
$$

on itself at the boxed position of

$$
f(y) \simeq f g f(y)
$$

where the most general unifier of $f f(x)$ and $f(y)$ is $\{y \mapsto f(x)\}$, we get

$$
f f g f(x) \simeq f g f f(x) .
$$

Now, by renaming the variables, both sides of the conclusion

$$
f f g f(z) \simeq f g f f(z)
$$


can be rewritten in one step with $f f(x) \simeq f g f(x)$ (oriented with $\succ_{r}$ ) into $f g f g f(z)$, i.e., the conclusion of the inference can be simplified into a tautology. On the other hand, by an ordered paramodulation with

$$
f f(x) \simeq f g f(x)
$$

on itself at the boxed position of

$$
f f(y) \simeq f g f(y)
$$

we get

$$
f f f(x) \simeq f g f g f(x),
$$

i.e., $f f f(z) \simeq f g f g f(z)$. In this case, $f f f(z)$ can be rewritten in two steps with $f f(x) \simeq$ $f g f(x)$ (oriented with $\succ_{r}$ ) into $f g f g f(z)$, and hence the conclusion of the inference can be simplified into a tautology as before.

Unfortunately, the system is not saturated. This is because, according to our requirements, the variable $x$ of the rewrite rule $f f(x) \rightarrow f g f(x)$ used to simplify has to be marked if it has been instantiated with a non-variable term when rewriting, as it happens here. Hence, the equation $f f(z) \cdot\{z \mapsto x\} \simeq f g f(z) \cdot\{z \mapsto x\}$ must be added. This equation subsumes the original one $f f(x) \simeq f g f(x)$. However, we must consider inferences with the new equation, leading to some new equations which cannot be simplified at all, and the process does not terminate. For instance, the inference with

$$
f f(z) \cdot\{z \mapsto x\} \simeq f g f(z) \cdot\{z \mapsto x\}
$$

on itself at the boxed position of

$$
f(z) \cdot\{z \mapsto y\} \simeq f g f(z) \cdot\{z \mapsto y\},
$$

with $\sigma=\operatorname{mgu}(f f(z)\{z \mapsto x\}, f(z)\{z \mapsto y\})=\operatorname{mgu}(f f(x), f(y))=\{y \mapsto f(x)\}$, gives us

$$
f\left(z^{\prime}\right) \cdot\left\{z^{\prime} \mapsto f g f(x)\right\} \simeq f g f(z) \cdot\{z \mapsto f(x)\},
$$

i.e., we mark the inference position according to case $4 \mathrm{c}$ of the inference system given in Section 4.1 (since $f f(z) \nsucc f f g f(x)$ and $f(z) \cdot\{z \mapsto f(x)\} \ngtr_{m} f g f(z) \cdot\{z \mapsto x\}$ ). Notice that by adding a mark at the inference position we guarantee a decrease w.r.t. $>_{m}$ in the skeleton of the left-hand side. Since, moreover, we keep the marks of the right-hand side of the premise, the conclusion obtained is smaller. However, due to the marks, the conclusion of the inference cannot be simplified at all with the marked rule $f f(z) \cdot\{z \mapsto x\} \rightarrow f g f(z) \cdot\{z \mapsto x\}$ (notice that $f f(z)$ matches neither $f\left(z^{\prime}\right)$ nor $f g f(z)$ ). But, in spite of this necessary protection against simplification, inferences are necessary even below marks and, unfortunately, this makes this saturation diverge.

In conclusion, from our experimentation it follows that the ideas presented in this paper can be easily implemented and work reasonably well for small examples. However, as we have seen, the amount of simplification we can use is still not enough to capture known examples for which canonical rewrite system exist and our procedure diverges. Altogether, we believe that the results are good enough to consider them as a reasonable way to add redundancy notions in cases where using non-monotonic orderings is mandatory, like for instance when working modulo equational theories (as explained in the introduction). 


\section{Building West Orderings}

There are some known ways to build west orderings that are not monotonic. Note that weakly monotonic orderings like general linear polynomial interpretations (see, e.g., Baader and Nipkow (1998)) or the recursive path ordering (Dershowitz (1982)) with argument filterings (Arts and Giesl (2000); Kusakari et al (1999)) cannot be used, because they do not fulfill the subterm property. A well-known method that produces a west ordering is the semantic path ordering (Kamin and Levy (1980)). There, the non-monotonic ordering is built on top of a well-founded underlying base ordering $>_{B}$ and an equivalence relation $=_{B}$ compatible with $>_{B}$ as follows.

Definition 25 Let $\succ_{B}$ be an ordering on terms, and $s$ and $t$ be two terms. Then, $\succ_{S P O}$ is defined as $s=f\left(s_{1}, \ldots, s_{n}\right) \succ_{S P O} t$ if and only if

1. $s_{i} \succeq_{S P O} t$ for some $i \in\{1 \ldots n\}$;

2. $t=g\left(t_{1}, \ldots, t_{m}\right), s>_{B} t$ and $s \succ_{S P O} t_{i}$ for al $i \in\{1 \ldots m\}$;

3. $t=g\left(t_{1}, \ldots, t_{m}\right), s={ }_{B} t$ and $\left\langle s_{1}, \ldots, s_{n}\right\rangle\left(\succ_{S P O}\right)_{l e x}\left\langle t_{1}, \ldots, t_{m}\right\rangle$.

The Semantic Path Ordering is well-founded if the base ordering $>_{B}$ is well-founded. It is total on ground terms if $\succ_{B} \cup={ }_{B}$ also is, and case 1 ensures that the ordering includes the subterm relation. On the other hand, due to this case, even if $>_{B}$ is (weakly) monotonic the resulting semantic path ordering may not be monotonic.

Still, we have monotonicity for some comparisons, and a monotonic subrelation of a given SPO can be extracted. This is exploited in the method called the Monotonic Semantic Path Ordering, (Borralleras et al (2000)), MSPO for short, which can be used to define the monotonic part of the SPO, and hence a reduction ordering inside the west ordering. However, it is not easy to build an ordering like the one used in Example 2, i.e. including $a \succ b, f(a) \succ f(b), g(b) \succ g(a)$, with standard base orderings.

In what follows, we will define a class of base orderings $>_{B}$ which can be used to obtain an SPO including most of our examples.

Assume we have a linear polynomial interpretation $I$ that associates a linear polynomial $I_{f}$ on non-negative integers to every function symbol $f$ in the signature $\mathscr{F}$. Then $I(t)$ is defined as $t$ if $t$ is a variable and as $I_{f}\left(I\left(t_{1}\right), \ldots, I\left(t_{n}\right)\right)$ if $t=f\left(t_{1}, \ldots, t_{n}\right)$. Additionally we have a subset $\mathscr{F} P$ of the set of symbols $\mathscr{F}$ totally ordered by a well-founded precedence $\succ_{\mathscr{F}}$. Then we define the ordering as follows.

Definition 26 Let $s$ and $t$ be two terms. Then $s=f\left(s_{1}, \ldots, s_{n}\right)>_{B} g\left(t_{1}, \ldots, t_{m}\right)=t$ if and only if

1. $f, g \in \mathscr{F}_{P}, f \succ \mathscr{F} g$;

2. $f \notin \mathscr{F}_{P}, g \in \mathscr{F}_{P}$;

3. $f, g \notin \mathscr{F}_{P}$ and $I(s)>I(t)$.

Similarly $s=f\left(s_{1}, \ldots, s_{n}\right)={ }_{B} g\left(t_{1}, \ldots, t_{m}\right)=t$ if and only if

1. $f, g \in \mathscr{F}_{P}, f=g$

2. $f, g \notin \mathscr{F}_{P}$ and $I(s)=I(t)$.

The ordering is trivially well-founded, since the precedence is well-founded and the polynomial interpretation only uses non-negative integers. Note that case 2 can as well be written the other way round, i.e. with $f \in \mathscr{F}_{P}, g \notin \mathscr{F}_{P}$, preserving well-foundedness. Finally, 
totality on ground terms of $>_{B} \cup={ }_{B}$ and compatibility of $>_{B}$ and $=_{B}$ is straightforward as well (recall that $\succ_{\mathscr{F}}$ is assumed to be total on $\mathscr{F}_{P}$ ).

Moreover, following the ideas of the MSPO, we can easily show that for all terms $s$ and $t$ such that $I(s) \geq I(t)$ we have that $s \succ_{S P O} t$ implies $u[s] \succ_{S P O} u[t]$, i.e. the reduction ordering $\succ_{r}$ included in $\succ_{S P O}$ fulfills that $s \succ_{S P O} t$ and $I(s) \geq I(t)$ implies $s \succ_{r} t$.

Lemma 22 Let $s$ and $t$ be terms. If $I(s) \geq I(t)$ and $s \succ_{S P O} t$ then $u[s] \succ_{S P O} u[t]$.

Proof We proceed by induction on the size of $u$. First note that, since $I(s) \geq I(t)$ we have that $I(u[s]) \geq I(u[t])$ for all non empty contexts $u$, and hence, we have either $u[s]={ }_{B} u[t]$ or $u[s]>_{B} u[t]$. Now, if $u$ is empty it holds. Otherwise, let $u[]=h\left(v_{1}, \ldots, u^{\prime}[], \ldots, v_{n}\right)$.

- If $u[s]={ }_{B} u[t]$ we apply case 3 of SPO and, by induction, $\left\langle v_{1}, \ldots, u^{\prime}[s], \ldots, v_{n}\right\rangle\left(\succ_{S P O}\right.$ )$_{l e x}\left\langle v_{1}, \ldots, u^{\prime}[t], \ldots, v_{n}\right\rangle$ holds.

- If $u[s]>_{B} u[t]$ we apply case 2 . Then, by case 1 , we have $u[s] \succ_{S P O} v_{j}$ for all $j$ and, by case 1 and induction hypothesis, we have $u[s] \succ_{S P O} u^{\prime}[t]$.

Let us show that, using this base ordering, SPO includes the ordering we have used in Example 2.

Example 8 In this example $\mathscr{F}=\{f, g, a, b\}$. Then we take $\mathscr{F}_{P}=\{f, a, b\}$ and $f \succ_{\mathscr{F}} a \succ_{\mathscr{F}}$ $b$. The polynomial interpretation is defined by $I_{f}(x)=0, I_{g}(x)=x, I_{a}=0$ and $I_{b}=1$. Let $\succ_{r}$ be the monotonic subrelation of $\succ_{S P O}$. We have to show that $a \succ_{S P O} b, f(a) \succ_{r} f(b)$ and $g(b) \succ_{r} g(a)$.

1. $a \succ_{S P O} b$ follows by case 2 of SPO applying case 1 of $>_{B}$.

2. $f(a) \succ_{\text {SPO }} f(b)$ follows by case 3 , since $f(a)=_{B} f(b)$ by case 1 . To conclude, since $I(f(a))=0=I(f(b))$, we have $f(a) \succ_{r} f(b)$ by Lemma 22 .

3. $g(b) \succ_{S P O} g(a)$ follows by case 2 of SPO, applying case 3 of $>_{B}$. Then, we need $g(b) \succ_{S P O} a$, which follows by case 2 of SPO applying case 2 of $>_{B}$. Finally, since $I(g(b))=1>0=I(g(a))$, we have $g(b) \succ_{r} g(a)$ by Lemma 22.

\section{Conclusion}

In this paper we have improved on the results of Bofill et al (2003) on completeness of ordered paramodulation with non-monotonic orderings, by adapting those refutation complete inference systems so that they are compatible with powerful redundancy elimination techniques such as demodulation and, hence, making them more likely to be used in practice. The main interest on considering non-monotonic orderings is to broaden the class of orderings that can be used in paramodulation, and especially when working modulo an equational theory.

We have proposed some inference systems (for equations and general first order clauses) that work with a pair of orderings: a west ordering $\succ$, which is used when performing inferences, and a reduction ordering $\succ_{r}$, included in $\succ$, which is used for applying simplification by rewriting. Our method is based on adding some marks that block terms for redundancy (but not for inferences). We have given a counterexample (Example 6) to refutation completeness of ordered paramodulation when dealing with a non-monotonic ordering $\succ$ and 
applying simplification by rewriting w.r.t. a reduction ordering $\succ_{r}$ included in $\succ$, if no marks at all are introduced.

In our calculus, the more equations can be handled by the (non-total) reduction ordering $\succ_{r}$ at hand, the less marks will be introduced in the conclusions of the inferences. In fact, if all equations can be handled by the reduction ordering, then no (new) marks will be introduced in the conclusion of any inference (notice that in this situation case 4a of the paramodulation inference rule always applies). However, is spite of this, we may have to introduce some marks in an equation (or clause) when using it for subsuming or simplifying other equations (or clauses). As stated in Section 5.5, in our notions of redundancy it is necessary that non-marking variables occurring in the skeleton of a marked term are only instantiated with variables when performing redundancy. Hence, in order to be able to perform redundancy sometimes we need to mark some variables (so that those variables become marking variables in the skeleton). This addition of marks guarantees refutation completeness but, since new inferences are necessary with the marked version of the equation, this may sometimes prevent the procedure from saturating the set of clauses (as shown in Example 7).

Using these results in the same way as in Bofill et al (2003), we have obtained a KnuthBendix completion procedure, compatible with simplification, that finds a convergent TRS for a given set of equations $E$ and a (possibly non-totalizable) reduction ordering $\succ_{r}$ whenever it exists. Our results on Knuth-Bendix completion could also be extended in the line of the results on completion with termination tools in Wehrman et al (2006); Winkler and Middeldorp (2010). There, reduction orderings are replaced by termination tools. Since nowadays most state-of-the-art termination tools use both monotonic and non-monotonic orderings, we believe that using termination tools in our framework for automatically generating the monotonic and non-monotonic orderings on demand can increase the applicability of our results. Note that, in our case fixing in advance the non-monotonic ordering and the reduction ordering included in it (using techniques like the ones given in Section 8) is even harder than in the standard case, where only a (total) reduction ordering is needed.

A prototype implementation of our results for the equational case has shown that, in some small examples, the allowed simplification suffices to obtain a complete term rewrite system.

\section{References}

Arts T, Giesl J (2000) Termination of term rewriting using dependency pairs. Theoretical Computer Science 236:133-178

Baader F, Nipkow T (1998) Term rewriting and all that. Cambridge University Press, New York, NY, USA

Bachmair L, Dershowitz N (1994) Equational inference, canonical proofs, and proof orderings. Journal of the ACM 41(2):236-276

Bachmair L, Ganzinger H (1994) Rewrite-based equational theorem proving with selection and simplification. Journal of Logic and Computation 4(3):217-247

Bachmair L, Ganzinger H (1998) Equational reasoning in saturation-based theorem proving. In: Bibel W, Schmitt P (eds) Automated Deduction - A Basis for Applications, vol I, Kluwer, Dordrecht, The Netherlands, chap 11, pp 353-397

Bachmair L, Dershowitz N, Hsiang J (1986) Orderings for equational proofs. In: First IEEE Symposium on Logic in Computer Science (LICS), IEEE Computer Society Press, Los Alamitos, CA, USA, Cambridge, MA, USA, pp 346-357

Bachmair L, Dershowitz N, Plaisted D (1989) Completion Without Failure. In: Aït-Kaci H, Nivat M (eds) Resolution of Equations in Algebraic Structures, vol 2: Rewriting Techniques, Academic Press, New York, chap 1, pp 1-30

Bachmair L, Ganzinger H, Lynch C, Snyder W (1995) Basic paramodulation. Information and Computation 121(2):172-192 
Bofill M, Rubio A (2002) Well-foundedness is sufficient for completeness of ordered paramodulation. In: 18th International Conference on Automated Deduction (CADE), Springer-Verlag, Berlin Heidelberg, Germany, Copenhagen, Denmark, LNAI, vol 2392, pp 456-470

Bofill M, Rubio A (2004) Redundancy notions for paramodulation with non-monotonic orderings. In: 2nd International Joint Conference on Automated Reasoning (IJCAR), Springer-Verlag, Berlin Heidelberg, Germany, Cork, Ireland, LNAI, vol 3097, pp 107-121

Bofill M, Rubio A (2009) Paramodulation with well-founded orderings. Journal of Logic and Computation 19(2):263-302

Bofill M, Godoy G, Nieuwenhuis R, Rubio A (1999) Paramodulation with non-monotonic orderings. In: 14th IEEE Symposium on Logic in Computer Science (LICS), IEEE Computer Society Press, Los Alamitos, CA, USA, Trento, Italy, pp 225-233

Bofill M, Godoy G, Nieuwenhuis R, Rubio A (2003) Paramodulation and Knuth-Bendix Completion with Nontotal and Nonmonotonic Orderings. Journal of Automated Reasoning 30(1):99-120

Borralleras C, Ferreira M, Rubio A (2000) Complete monotonic semantic path orderings. In: McAllester D (ed) Proceedings of the 17th International Conference on Automated Deduction (CADE-17), SpringerVerlag, Pittsburgh, USA, LNAI, vol 1831, pp 346-364

Dershowitz N (1982) Orderings for term-rewriting systems. Theoretical Computer Science 17(3):279-301

Dershowitz N, Jouannaud JP (1990) Rewrite systems. In: van Leeuwen J (ed) Handbook of Theoretical Computer Science, vol B: Formal Models and Semantics, Elsevier Science B.V, Amsterdan, The Netherlands and The MIT Press, Cambridge, MA, USA, chap 6, pp 244-320

Dershowitz N, Manna Z (1979) Proving termination with multiset orderings. Communications of the ACM 22(8), pp 465-476

Devie H (1990) Linear completion. In: Kaplan S, Okada M (eds) Conditional and Typed Rewriting Systems, 2nd International Workshop, Springer-Verlag, Montreal, Canada, LNCS 516, pp 233-245

Hsiang J, Rusinowitch M (1991) Proving refutational completeness of theorem proving strategies: the transfinite semantic tree method. Journal of the ACM 38(3):559-587

Kamin S, Levy JJ (1980) Two generalizations of the recursive path ordering, unpublished note, Dept. of Computer Science, Univ. of Illinois, Urbana, IL

Kusakari K, Nakamura M, Toyama Y (1999) Argument filtering transformation. In: Proceedings of the International Conference on Principles and Practice of Declarative Programming (PPDP'99), SpringerVerlag, LNCS 1702, pp 47-61

Nieuwenhuis R, Rubio A (1995) Theorem Proving with Ordering and Equality Constrained Clauses. Journal of Symbolic Computation 19(4):321-351

Nieuwenhuis R, Rubio A (2001) Paramodulation-based theorem proving. In: Robinson J, Voronkov A (eds) Handbook of Automated Reasoning, vol 1, Elsevier Science B.V, Amsterdan, The Netherlands and The MIT Press, Cambridge, MA, USA, chap 7, pp 372-444

Wechler W (1992) Universal Algebra for Computer Scientists, EATCS Monographs on Theoretical Computer Science, vol 25. Springer-Verlag, New York

Wehrman I, Stump A, Westbrook EM (2006) Slothrop: Knuth-Bendix completion with a modern termination checker. In: Pfenning F (ed) RTA, Springer, Lecture Notes in Computer Science, vol 4098, pp 287-296

Winkler S, Middeldorp A (2010) Termination tools in ordered completion. In: Proceedings of the 5th International Joint Conference on Automated Reasoning, Springer-Verlag, Lecture Notes in Artificial Intelligence, vol 6173, pp 518-532 